\title{
CircSPIDR acts as a tumour suppressor in cervical adenocarcinoma by sponging miR-431-5p and regulating SORCS1 and CUBN expression
}

\author{
Junfen $\mathrm{Xu}^{1}$, Weiguo $\mathrm{Lu}^{1,2,3}$ \\ ${ }^{1}$ Department of Gynecologic Oncology, Women's Hospital, Zhejiang University School of Medicine, Hangzhou \\ 310006, Zhejiang, China \\ ${ }^{2}$ Center of Uterine Cancer Diagnosis \& Therapy of Zhejiang Province, Hangzhou 310006, Zhejiang, China \\ ${ }^{3}$ Zhejiang University Cancer Center, Hangzhou 310006, Zhejiang, China
}

Correspondence to: Weiguo Lu; email: lbwg@zju.edu.cn

Keywords: CircSPIDR, cervical adenocarcinoma, miR-431-5p, SORCS1, CUBN, cell growth

Received: February 8, 2021 Accepted: June 19, $2021 \quad$ Published: July 29, 2021

Copyright: (c) $2021 \mathrm{Xu}$ and Lu. This is an open access article distributed under the terms of the Creative Commons Attribution License (CC BY 3.0), which permits unrestricted use, distribution, and reproduction in any medium, provided the original author and source are credited.

\section{ABSTRACT}

To identify circular RNAs (circRNAs) with tumor suppressor activity against cervical adenocarcinoma, we compared the circRNA levels of cervical adenocarcinoma and normal cervical tissues. We found that circSPIDR was dramatically downregulated in cervical adenocarcinoma tissues. In cervical adenocarcinoma cells, overexpression of circSPIDR reduced cell viability, inhibited colony formation and promoted apoptosis, whereas knockdown of circSPIDR exerted the opposite effects. CircSPIDR overexpression also suppressed the tumorigenicity of cervical adenocarcinoma cells in a xenograft mouse model. CircSPIDR was found to sponge miR-431-5p, thereby de-repressing sortin-related VPS10 domain-containing receptor 1 (SORCS1) and cubilin (CUBN) and inhibiting the development of cervical adenocarcinoma. In clinical cervical samples, circSPIDR expression correlated negatively with miR-431-5p expression and positively with SORCS1 and CUBN expression. These results demonstrated that circSPIDR suppresses cervical adenocarcinoma by competitively binding to miR-431-5p, thus upregulating SORCS1 and CUBN. These findings suggest circSPIDR could serve as a novel therapeutic target for treatment of cervical adenocarcinoma patients.

\section{INTRODUCTION}

Cervical cancer is the fourth most common cancer and the fourth leading cause of cancer death in women worldwide [1]. In China, approximately 98,900 new cervical cancer cases were diagnosed and 30,500 women died of this disease in 2015 [2]. Overall, cervical squamous cell carcinoma is the primary pathological type of human cervical cancer, while cervical adenocarcinoma (CADC) is the second. In recent decades, the tumor screenings and human papillomavirus vaccination programs have greatly reduced the burden of cervical squamous cell carcinoma in developed countries; however, the incidence of CADC has increased during the same period worldwide $[2,3]$. Thus, it is critical to determine the underlying molecular mechanisms of CADC and develop effective therapies.

Circular RNAs (circRNAs) are regulatory RNAs characterized by covalent single-stranded loop structures generated via back-splicing or exon skipping of the precursor mRNA [4-7]. CircRNAs are naturally resistant to RNA exonucleases, are tissue- or cell typespecific $[5,8]$, and exert important functions in various human cancers $[9,10]$. To date, circRNAs have largely been reported to serve as competing endogenous RNAs (ceRNAs). For example, circTP63 (an oncogene in lung squamous cell carcinoma), circ-ZKSCAN1 (a bladder cancer suppressor) and other cancer-related circRNAs such as CDR1as [11-13] and circAKT3 [14, 15] function as microRNA (miRNA) sponges that inhibit the effects 
of miRNAs on their target genes. In addition to the ceRNA mechanism, circRNAs can sequester specific RNA-binding proteins to regulate gene expression [4, 16], and some of them can be translated into functional proteins [17-19]. Certain circRNAs have been identified as promising clinical molecular biomarkers for cancer diagnosis and treatment [20-22]. However, the functions of circRNAs in CADC remain to be elucidated.

In the present study, our main interest was to identify circRNAs with tumor suppressor functions in CADC. In a previous study using RNA-sequencing data from human CADC and normal cervical tissues (GSE No. 145372), we found that one of the most significantly downregulated circRNAs in CADC tissues was circSPIDR, - a circRNA derived from exons 6 and 7 of the human scaffold protein involved in DNA repair (SPIDR) gene. Thus, in the current study, we evaluated the effects of circSPIDR on cell proliferation, colony formation and apoptosis in CADC cells. Then, we examined the influence of circSPIDR on miRNAs and their target genes, and determined the effects of these genes on the malignant behaviors of CADC cells. Finally, we assessed the clinical importance of circSPIDR expression by evaluating its correlation with miRNA and target gene expression in a large cohort of primary CADC tissues. Our study suggested that circSPIDR is a tumor suppressor that may be a candidate for the diagnosis and treatment of CADC.

\section{RESULTS}

\section{CircSPIDR is significantly down-regulated in CADC}

We previously used transcriptome sequencing to determine the circRNA expression signatures of CADC patients [23], and found that a novel circRNA named circSPIDR was significantly downregulated in CADC tissues compared with normal cervical tissues (Figure 1A). CircSPIDR is derived from exons 6 and 7 of the human SPIDR gene, and is 352 nucleotides long (Figure 1B). To confirm our circRNA sequencing data, we used quantitative real-time PCR (qRT-PCR) to assess circSPIDR expression in another 40 human cervical samples (20 normal vs. 20 CADC tissues). CircSPIDR expression was much lower in CADC tissues than in normal control tissues (Figure 1C).

\section{Characterization of circSPIDR in CADC cells}

Next, we used Sanger sequencing to assess the structure of circSPIDR (Figure 1B). Northern blotting confirmed that circSPIDR could be detected at approximately 352 nucleotides with a probe targeting the back-spliced junction in CADC cells (HeLa) and tissues (Figure 1D). A stability analysis of circSPIDR and SPIDR indicated that the loop structure of circSPIDR was resistant to digestion with RNase $\mathrm{R}$ exonuclease, while the linear SPIDR mRNA was degraded upon RNase $\mathrm{R}$ treatment (Figure 1E).

To determine whether head-to-tail splicing was the result of trans-splicing or genomic rearrangement, we designed divergent and convergent primers for circSPIDR. An RT-PCR analysis of reverse-transcribed RNA (cDNA) and genomic DNA (gDNA) from HeLa cells indicated that the divergent circSPIDR primers could amplify products from the cDNA, but not from the gDNA (Figure 1F). These results confirmed that circSPIDR is a stable circRNA expressed in CADC cells.

\section{CircSPIDR exerts tumor-suppressive effects in CADC cells in vitro and in vivo}

To evaluate the effects of circSPIDR on CADC cells, we conducted a circSPIDR expression vector and two specific siRNAs against circSPIDR. The overexpression plasmids and siRNAs were transfected into HeLa cells, and the cells were harvested for experiments after $48 \mathrm{~h}$. The circSPIDR vector successfully increased circSPIDR expression rather than SPIDR mRNA expression in HeLa cells (Supplementary Figure 1A). CircSPIDR siRNA \#1 and \#2 specifically inhibited circSPIDR expression without influencing SPIDR mRNA expression (Supplementary Figure 1B). These results also indicated that SPIDR expression was not altered by circSPIDR expression.

We then performed Cell Counting Kit 8 (CCK-8) and colony formation assays. Which demonstrated that overexpression of circSPIDR significantly suppressed $\mathrm{HeLa}$ cell viability and colony formation (Figure 2A and 2B). Conversely, knockdown of circSPIDR significantly enhanced cell viability and colony formation (Figure 2C and 2D). A bromodeoxyuridine (BrdU) incorporation assay indicated that the percentage of the BrdU-positive (proliferating) cells was lower in circSPIDR-overexpressing HeLa cells than in vector control cells (Supplementary Figure 2). Apoptosis assays illustrated that circSPIDR overexpression remarkably increased the proportion of apoptotic HeLa cells (Figure 2E), whereas circSPIDR knockdown had the opposite effect (Figure 2F). These results indicated that circSPIDR significantly suppressed cell proliferation and induced apoptosis in HeLa cells.

To identify the effects of circSPIDR in vivo, we stably expressed circSPIDR or an empty vector in HeLa cells 
using neomycin selection for 10 days. Then, we subcutaneously injected these cells into the right single flank of BALB/c nude mice ( $n=6 /$ group) to establish a xenograft tumor model. The tumors formed after one week, and the tumor size were monitored for the next five weeks. Tumors derived from circSPIDR overexpressing cells were much smaller than those derived from control cells (Figure $3 \mathrm{~A}$ and 3B), suggesting that circSPIDR significantly inhibited tumor growth. Immunohistochemical staining of the xenograft tumor tissues demonstrated that the proliferation marker Ki-67 was expressed at lower levels in the circSPIDRoverexpressing group (Figure 3C). In addition to generating the subcutaneous tumor model, we also intraperitoneally injected the two groups of cells into nude mice ( $n=5$ /group), and found that the tumors were much smaller in the circSPIDR group than in the vector control group (Figure 3D and 3E). These results
A

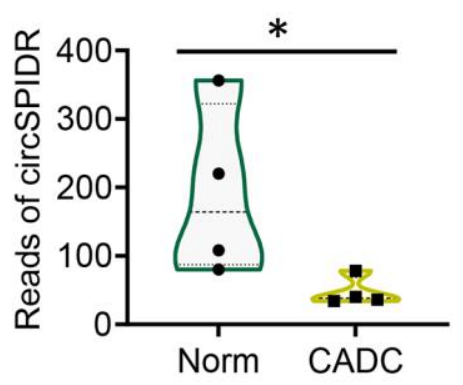

C

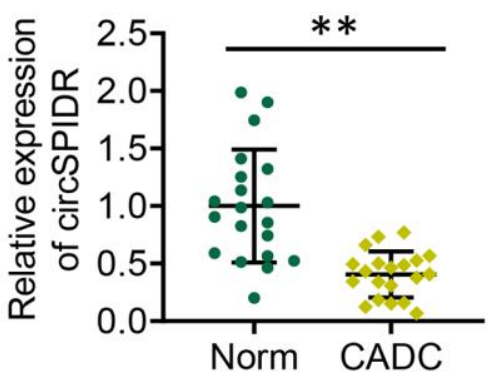

B
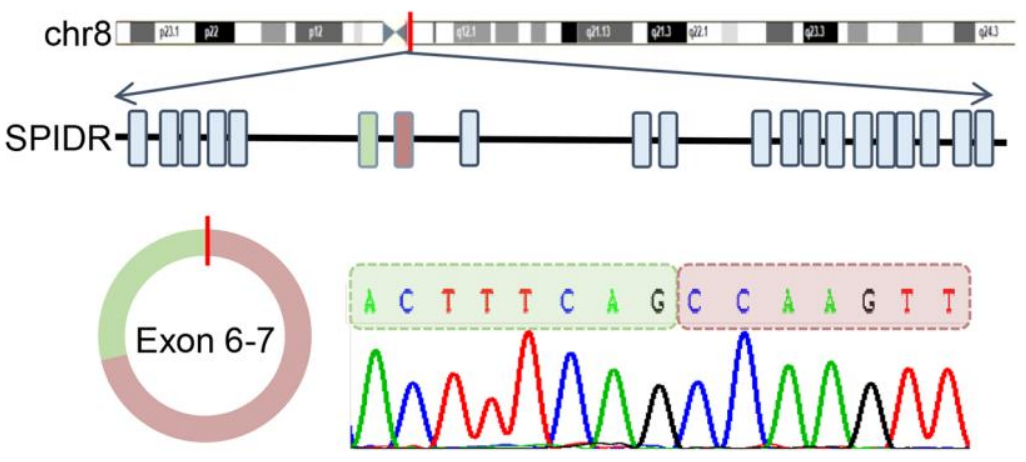

D

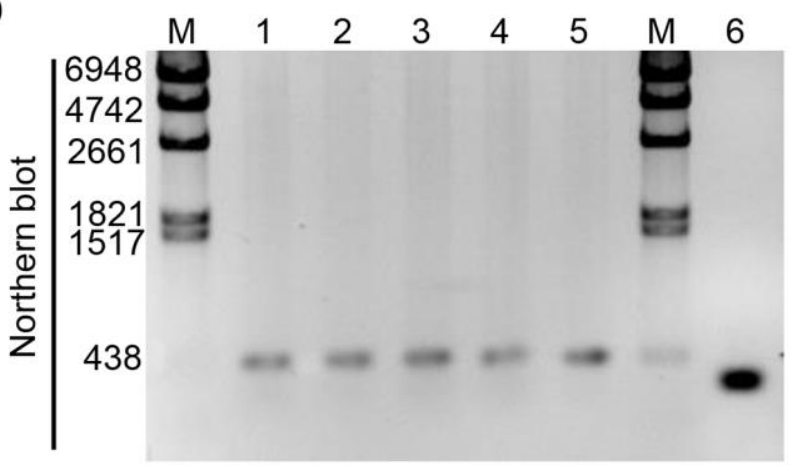

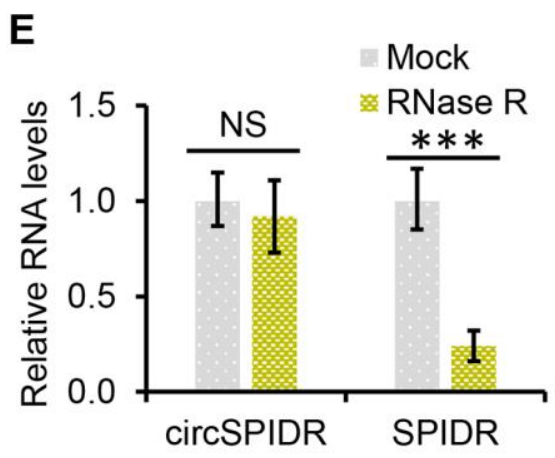
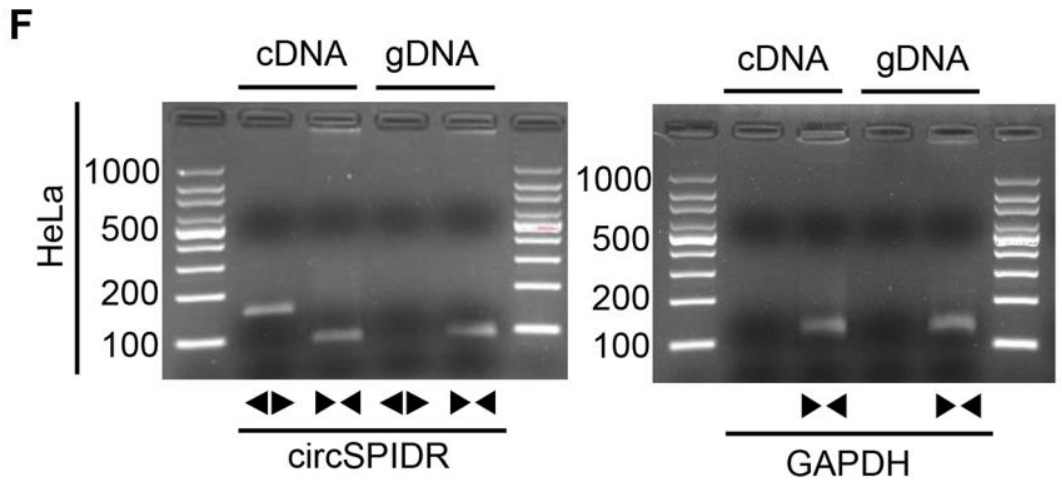

Figure 1. Characterization and validation of circSPIDR expression in CADC. (A) Violin plot showing the distribution of the RNA sequencing reads of circSPIDR in normal cervical tissues (Norm) and CADC tissues. (B) The genomic locus and generation of circSPIDR. CircSPIDR is produced from exons 6 and 7 of the human SPIDR gene. The back-splice junction sequence of circSPIDR was detected using Sanger sequencing. (C) qRT-PCR analysis of circSPIDR expression in 20 normal cervical tissues and 20 CADC tissues. (D) Northern blot of circSPIDR. Hybridization was performed with exon 6-7 junction probes. M, RNA marker; 1-3, HeLa cell repeats; 4, CADC tissue; 5, Normal cervical tissue; 6, RT-PCR products of probes. (E) qRT-PCR analysis of circSPIDR expression and SPIDR mRNA expression in HeLa cells with or without RNase R treatment. (F) RT-PCR products of circSPIDR and its linear isoform (SPIDR) in cDNA and gDNA from HeLa cells. GAPDH was used as a control. NS, not significant; ${ }^{*} P<0.05 ;{ }^{* *} P<0.01 ;{ }^{* * *} P<0.001$. 
suggested that circSPIDR suppressed CADC tumor growth in vitro and in vivo.

\section{CircSPIDR sponges miR-431-5p in CADC cells}

One major function of circRNAs is to sponge miRNAs [24]. To identify miRNAs that could be targets of circSPIDR, we searched the miRanda bioinformatics prediction database. Then, we compared the results with the differentially expressed miRNA signature of CADC patients from our previous miRNA sequencing data [25]. We found that circSPIDR possessed the conserved target site of miR-431-5p, and that miR-431-5p was upregulated in CADC tissues.

To test the hypothesis that circSPIDR sponges miR431-5p, we first examined miR-431-5p levels in the same 40 human cervical samples assayed for circSPIDR
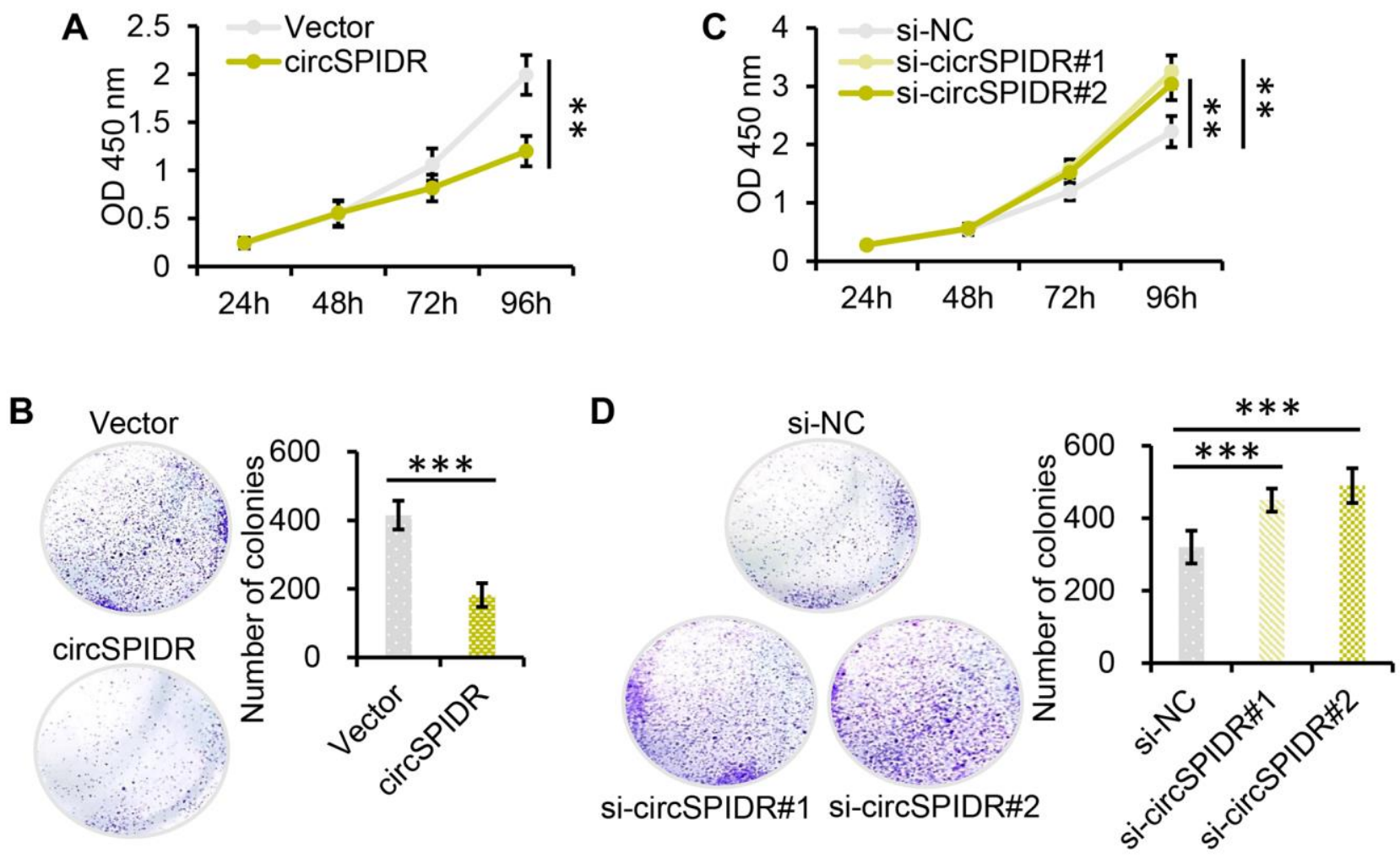
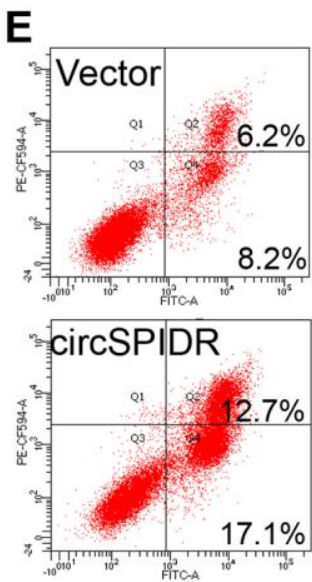

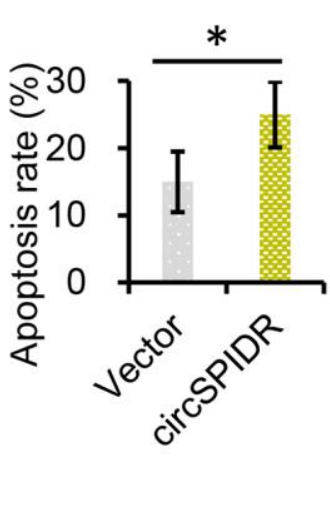

$\mathbf{F}$

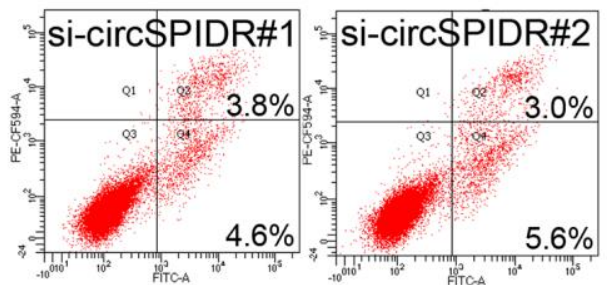

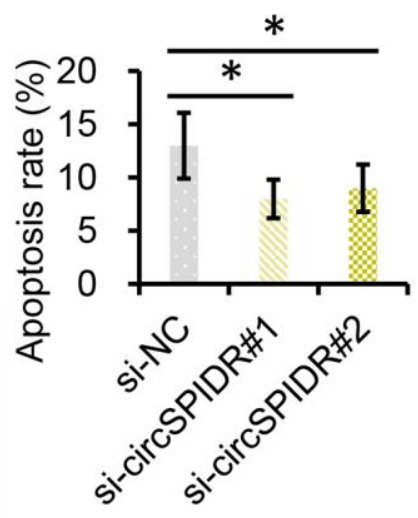

Figure 2. CircSPIDR inhibits cell growth and promotes apoptosis in CADC cells in vitro. (A, C) CCK-8 assays demonstrated that circSPIDR overexpression reduced HeLa cell viability (A), while circSPIDR inhibition with specific siRNAs (si-circSPIDR\#1 or si-circSPIDR\#2) increased HeLa cell viability (C). (B, D) Colony formation assays indicated that circSPIDR overexpression suppressed colony formation in HeLa cells (B), while circSPIDR inhibition promoted colony formation (D). (E, F) Representative images from flow cytometry analysis of apoptosis. Apoptotic cells were determined using Annexin V-FITC (X-axis) and PI (Y-axis) staining. CircSPIDR overexpression promoted HeLa cell apoptosis, while circSPIDR inhibition had the opposite effect. ${ }^{*} P<0.05 ;{ }^{* *} P<0.01 ;{ }^{* * *} P<0.001$. 
expression above. The qRT-PCR results revealed that miR-431-5p expression was significantly greater in CADC tissues with lower circSPIDR expression (Figure 4A). Next, we assessed the effects of circSPIDR expression on miR-431-5p expression in HeLa cells, and found that circSPIDR overexpression significantly reduced miR-431-5p expression, while circSPIDR knockdown increased miR-431-5p expression (Figure 4B). We then performed an RNA fluorescence in situ hybridization assay, which demonstrated that
A

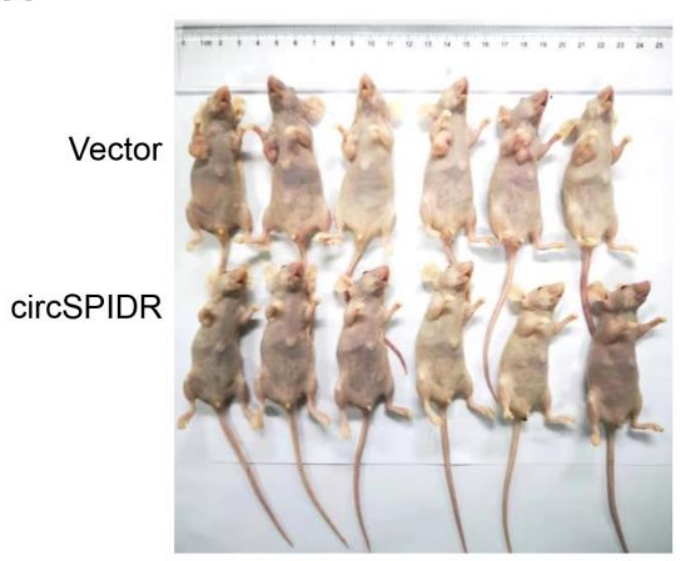

C

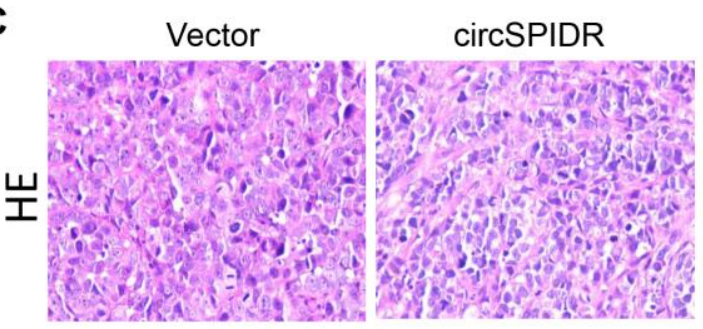

D

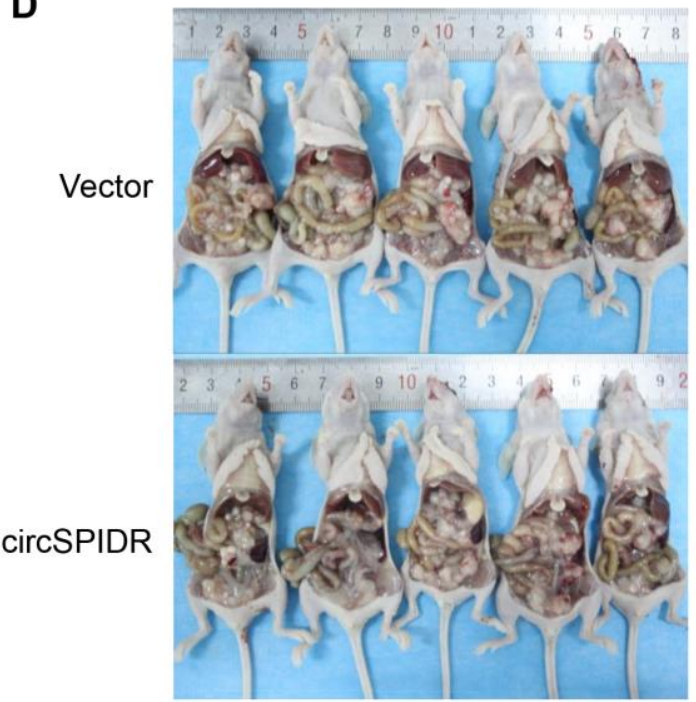

B
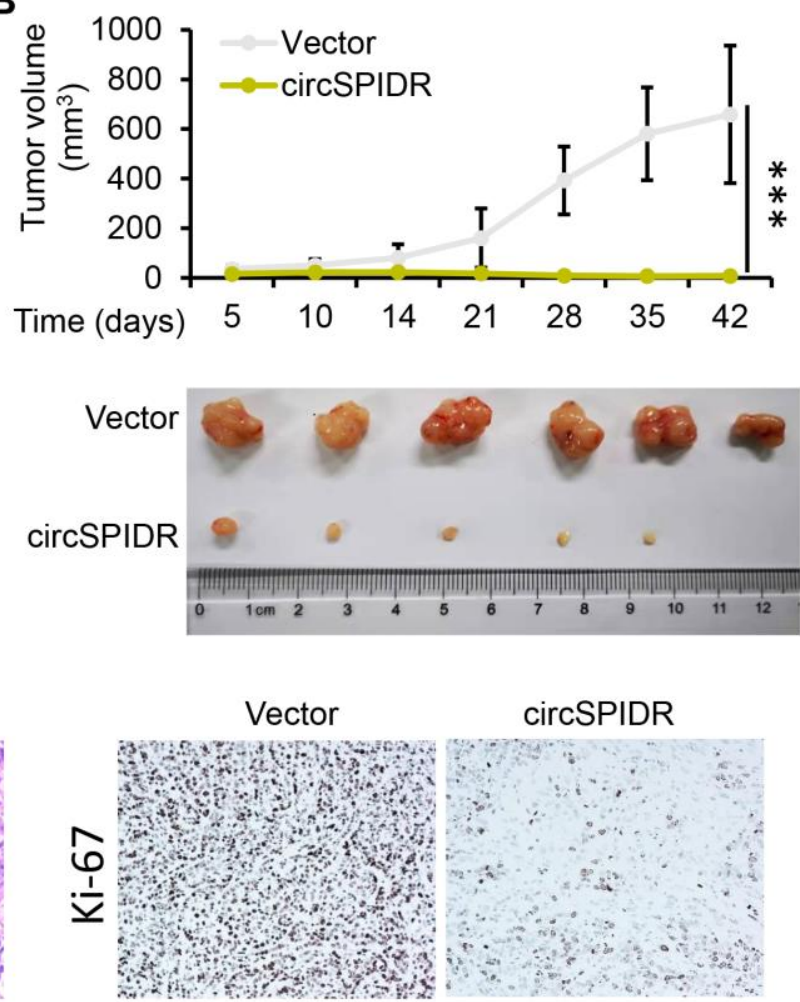

E
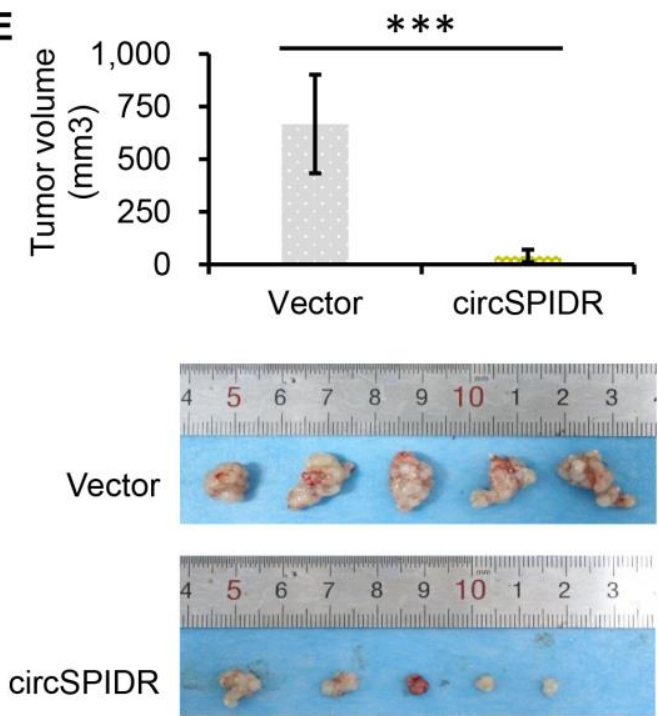

Figure 3. CircSPIDR overexpression inhibits HeLa cell growth in vivo. (A) HeLa cells expressing circSPIDR or the vector control were inoculated into BALB/c nude mice ( $n=6 /$ group) to establish subcutaneous xenograft tumors. Representative images of nude mice bearing CADC tumors are shown. (B) Growth curves and representative images of isolated xenograft tumours. The volume of the local tumors was measured. (C) Hematoxylin and eosin staining was performed and Ki-67 protein expression was evaluated in the xenograft tumors. (D, E) Representative images of nude mice intraperitoneally transplanted with HeLa/circSPIDR and HeLa/vector cells. ${ }^{* * *} P<0.001$. 
circSPIDR co-localized with miR-431-5p in HeLa cells (Figure 4C). Of note, circSPIDR and miR-431-5p were localized in both the cytoplasm and the nucleus, indicating that these RNAs may have additional functions aside from serving as ceRNAs.
Subsequently, we performed a dual luciferase reporter assay in 293T cells transfected with miR-431-5p mimics and luciferase vectors fused with circSPIDR wild-type (wt) or mutant (mut) promoters. As shown in Figure 4D, when miR-431-5p mimics were present,
A

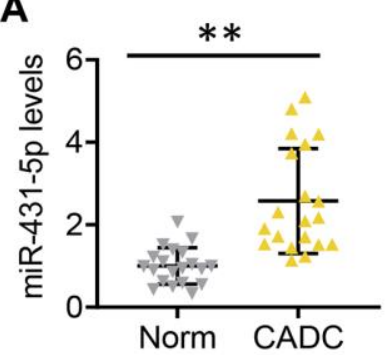

B

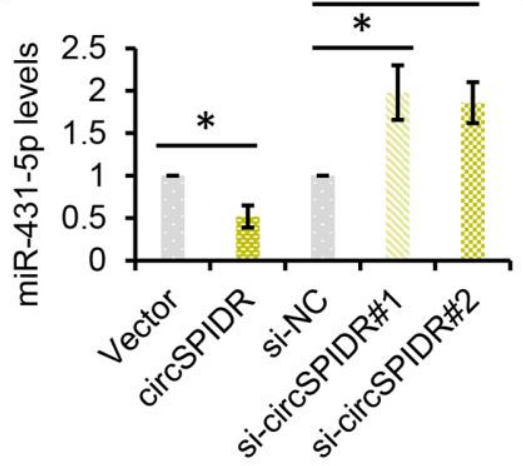

C

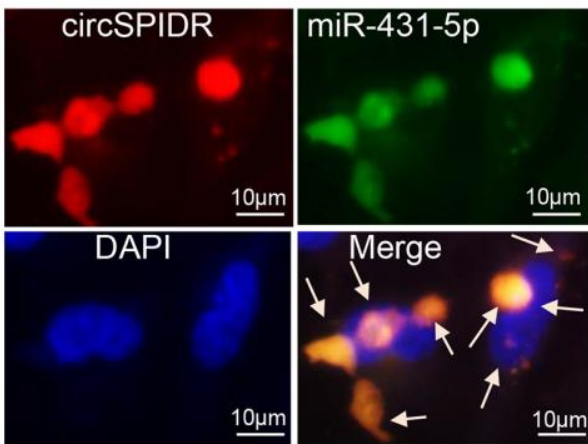

D

circSPIDR-wt: 5'...GGAUGCAAGACAGAUU....3'

IIIIIII

Has-miR-431-5p: 3'...GCCGGACGUUCUGU...5'

circSPIDR-mut: 5'...GGAU CGUUCUGUGAUU...3'

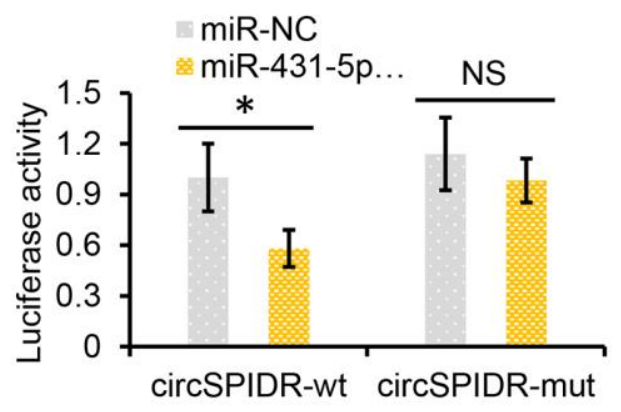

G
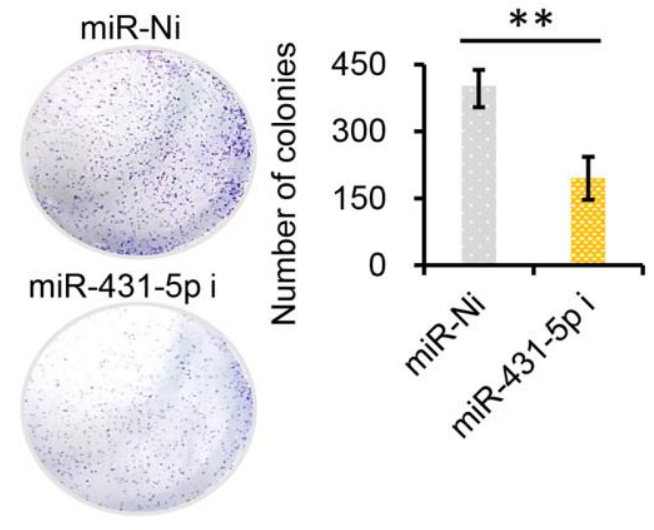

E

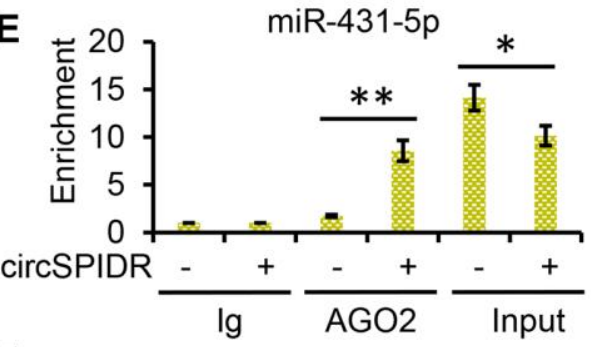

$\mathbf{F}$

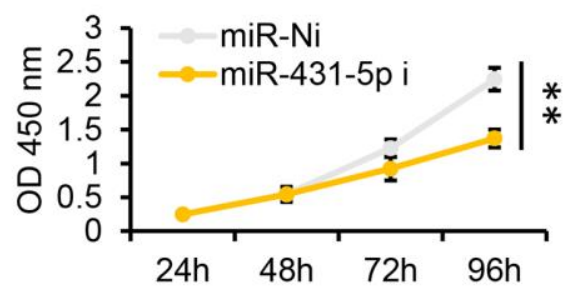

Figure 4. CircSPIDR directly binds to miR-431-5p as a miRNA sponge. (A) qRT-PCR analysis of miR-431-5p expression in 20 normal cervical tissues and 20 CADC tissues. (B) qRT-PCR analysis of miR-431-5p expression in HeLa cells transfected with circSPIDR, the vector, sicircSPIDR\#1, si-circSPIDR\#2 or si-NC. (C) RNA fluorescence in situ hybridization assay for circSPIDR and miR-431-5p in HeLa cells. (D) Schematic drawing showing the putative binding sites of miR-431-5p on circSPIDR. A luciferase reporter assay was performed to detect circSPIDR luciferase reporter activity in cells co-transfected with miR-431-5p mimics or miR-NC. (E) AGO2-RIP was conducted using an antiAGO2 antibody in HeLa cells transfected with circSPIDR or the vector. The enrichment of miR-431-5p was then assessed using qRT-PCR. (F-G) CCK-8 (F) and colony formation (G) assays of HeLa cells transfected with miR-431-5p inhibitors. (H) Apoptosis analysis of HeLa cells transfected with miR-431-5p inhibitors. NS, not significant; ${ }^{*} P<0.05 ;{ }^{* *} P<0.01$. 
luciferase reporter activity was significantly reduced in the circSPIDR-wt group, but not in the circSPIDR-mut group. In addition, we performed an RNA immunoprecipitation for argonaute 2 (AGO2-RIP) assay in circSPIDR-overexpressing and control HeLa cells. The results revealed that circSPIDR and miR-431$5 p$ were substantially enriched by AGO2 (Figure 4E). These findings indicated that circSPIDR could bind directly to $\mathrm{miR}-431-5 \mathrm{p}$.

To explore the function of miR-431-5p in CADC cells, we treated HeLa cells with inhibitors of miR-431-5p. CCK-8 and colony formation assays demonstrated that miR-431-5p inhibitors significantly reduced the growth of $\mathrm{HeLa}$ cells (Figure 4F and 4G). In addition, miR431-5p inhibitors promoted Hela cell apoptosis (Figure $4 \mathrm{H})$. Thus, miR-431-5p inhibition mimicked the phenotypic effects of circSPIDR overexpression in HeLa cells.
Next, we used miR-431-5p mimics to examine whether miR-431-5p overexpression could nullify the effects of circSPIDR overexpression in CADC cells. In rescue experiments, miR-431-5p overexpression reversed the circSPIDR-induced suppression of CADC cell proliferation and colony formation (Figure 5A and 5B). Co-transfection of circSPIDR with the miR-431-5p negative control (miR-NC) induced apoptosis in CADC cells, consistent with the effects of circSPIDR alone (see Figure 2E); however, miR-431-5p mimics impaired the apoptotic effects of circSPIDR (Figure 5C). These results demonstrated that miR-431-5p is the critical target of circSPIDR sponging activity in CADC.

\section{CircSPIDR relieves the repression of SORCSI and $C U B N$ by miR-431-5p in CADC cells}

Based on the ceRNA theory, we hypothesized that circSPIDR could enhance the expression of miR-431-5p
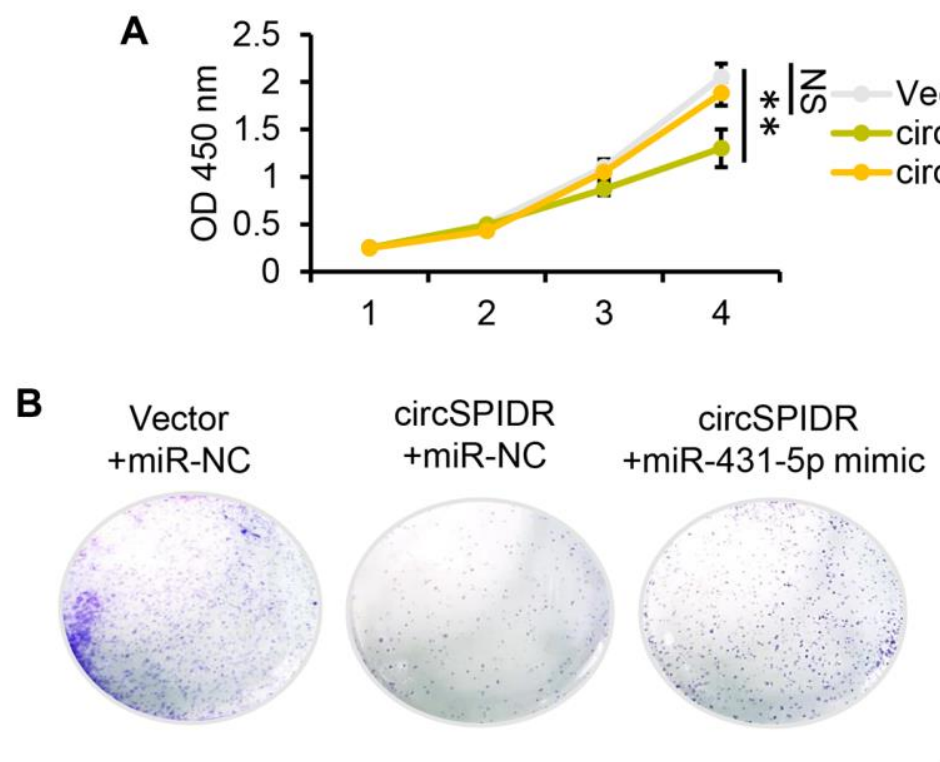

- Vector+miR-NC
- circSPIDR+miR-NC
- circSPIDR+miR-431-5p mimic

circSPIDR+miR-431-5p mimic
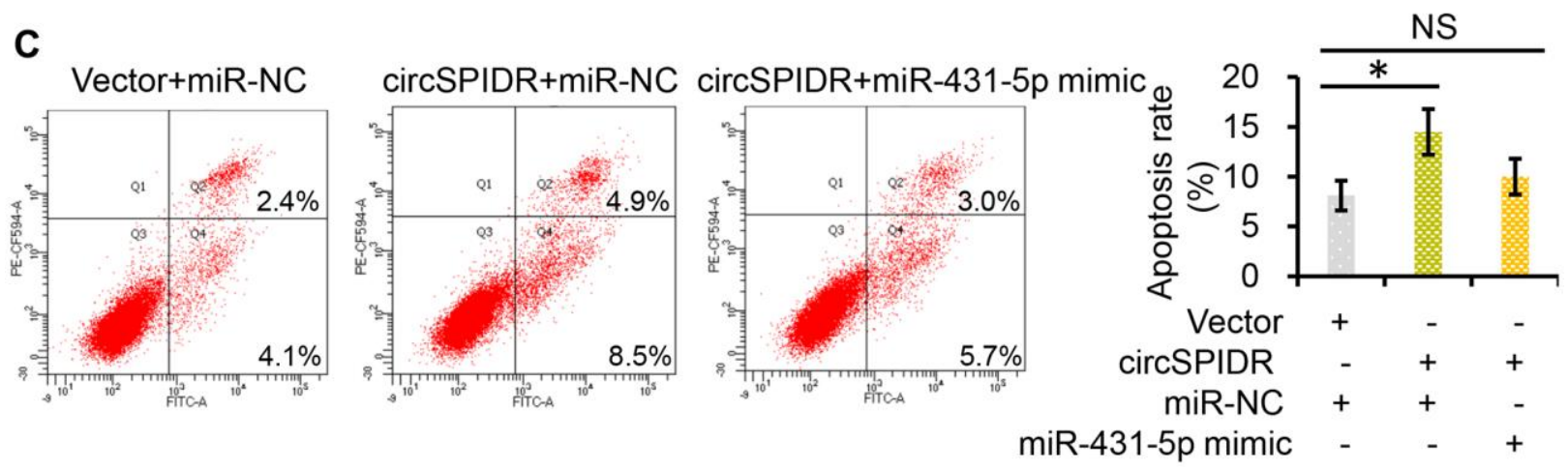

Figure 5. MiR-431-5p reverses the tumor-suppressive effects of circSPIDR in CADC cells. (A) CCK-8 assay evaluating the viability of HeLa cells co-transfected with circSPIDR and miR-431-5p mimics. (B) Colony formation assay in HeLa cells co-transfected with circSPIDR and miR-431-5p mimics. (C) Apoptosis analysis of HeLa cells co-transfected with circSPIDR and miR-431-5p mimics. NS, not significant; ${ }^{*} P<$ $0.05 ;{ }^{* *} P<0.01 ;{ }^{* * *} P<0.001$. 
target genes by sponging miR-431-5p. Using TargetScan and miRanda, we filtered the potential direct targets of miR-431-5p and mapped them to our previous transcriptome sequencing data for CADC [25]. We thus identified 16 putative miR-431-5p target genes that were significantly downregulated in CADC tissues: $A D D 2, C D H R 1, C U B N, D I R A S 2, E F C A B 1, K L F 8$,
L3MBT4, NXPH3, PLEKHG7, SCN3B, SHE, SORCS1, SOX5, TMEM213, WASF3 and ZNF483. (Figure 6A).

Next, we used miR-431-5p inhibitors to examine the effects of miR-431-5p on the expression of these genes in HeLa cells. Treatment of CADC cells with miR-431$5 p$ inhibitors strongly increased the expression of
A

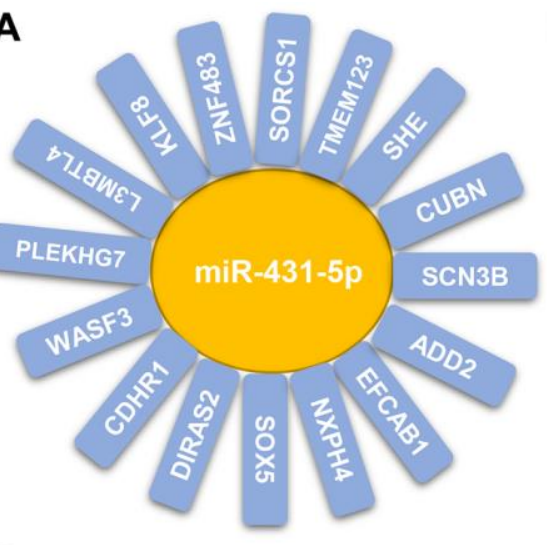

B

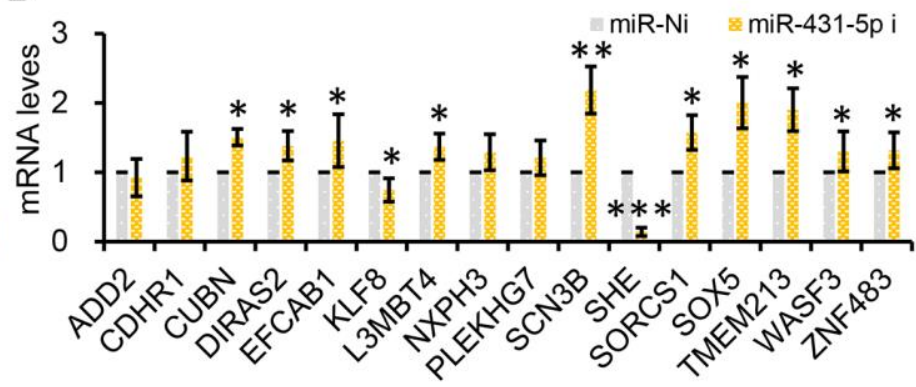

C

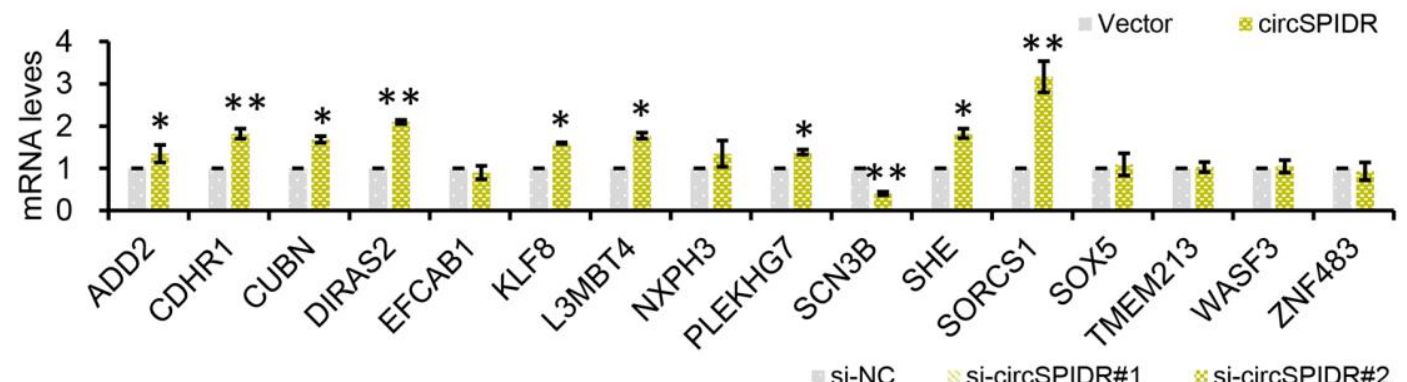

D

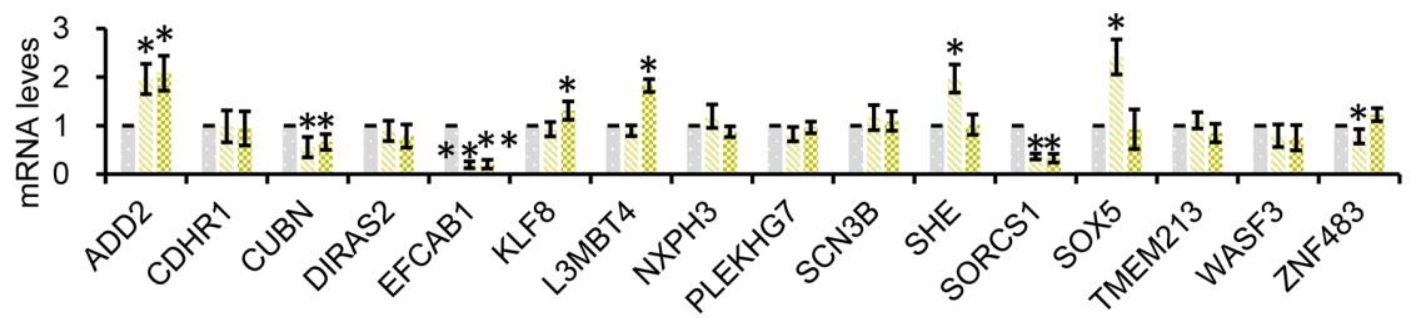

$\mathbf{E}$

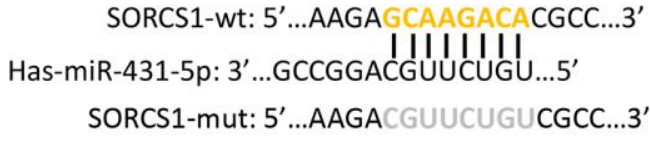

CUBN-wt: $5^{\prime}$...GACGGCAAGACUCCA...3'

Has-miR-431-5p: 3'...GCCGGACGUUCUGU...5

CUBN-mut: $5^{\prime}$...GACGCGUUCUGUCCA....3'

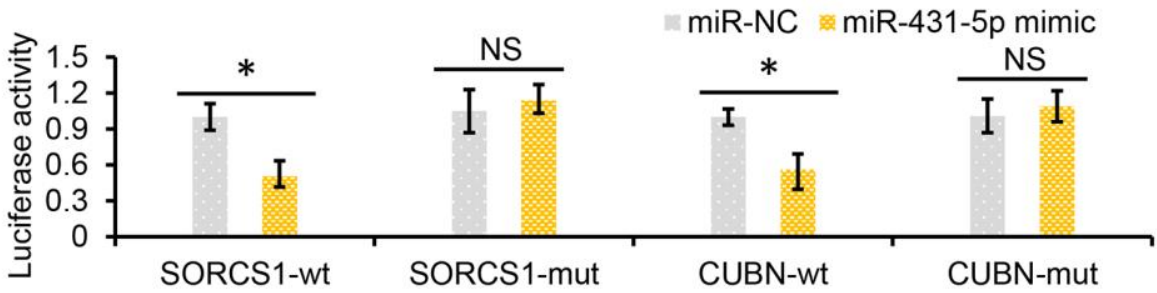

Figure 6. SORCS1 and CUBN are the target genes of miR-431-5p and circSPIDR. (A) miR-431-5p-target gene regulatory network. (B) qRT-PCR analysis of candidate target gene expression in CADC cells transfected with miR-431-5p inhibitors. (C, D) qRT-PCR analysis of candidate target gene expression in CADC cells transfected with the circSPIDR expression vector (C) or siRNAs against circSPIDR (D). (E) Luciferase activity of the $3^{\prime} U T R s$ of SORCS1 and the CUBN in 293T cells co-transfected with miR-431-5p mimics. NS, not significant; ${ }^{*} P<$ $0.05 ;{ }^{* *} P<0.01$. 
CUBN, DIRAS2, EFCAB1, L3MBT4, SCN3B, SORCS1, SOX5, TMEM213, WASF3 and ZNF483, but not the other genes (Figure 6B). However, overexpression of circSPIDR in HeLa cells only significantly upregulate $C U B N$ (cubilin) and SORCS1 (sortilin-related VPS10 domain-containing receptor 1), and knockdown of circSPIDR only significantly downregulated the same two genes (Figure 6C and 6D).

To verify that $S O R C S 1$ and $C U B N$ were the bona fide targets of miR-431-5p, we constructed SORCS1 3' untranslated region (UTR)-wt, SORCS1 3'UTR-mut, $C U B N$ 3'UTR-wt, and CUBN 3'UTR-mut luciferase reporter systems and co-transfected them with miR-431$5 p$ mimic into $293 \mathrm{~T}$ cells. The miR-431-5p mimics significantly reduced the activities of the luciferase reporter vectors carrying SORCS1 3'UTR-wt or CUBN $3^{\prime}$ UTR-wt sequences, but not of the vectors containing SORCS1 3'UTR- mut or CUBN 3'UTR- mut sequences (Figure 6E). Furthermore, inhibition of miR-431-5p markedly increased the protein levels of SORCS1 and CUBN in CADC cells (Figure 7A). Consistently, overexpression of circSPIDR significantly increased the protein levels of SORCS1 and CUBN in HeLa cells (Figure 7A).

To test whether SORCSI and CUBN were localized with the circSPIDR/miR-431-5p sponge complex, we performed an AGO2-RIP assay, which indicated that circSPIDR, miR-431-5p, SORCS1 and CUBN were mainly enriched with AGO2 (Figures 4E, 7B). In addition, the up-regulation of SORCS1 and CUBN in circSPIDR-overexpressing CADC cells could be partially reversed by co-transfection with miR-431-5p mimics (Figure 7C). Therefore, we considered SORCS1 and $C U B N$ as the major downstream targets of miR431-5p and circSPIDR.

Next, we assessed the involvement of SORCS1 and CUBN in circSPIDR/miR-431-5p signaling. The knockdown of SORCSI or CUBN using specific siRNAs significantly promoted cell survival and reduced apoptosis in CADC cells (Figure 7D, Supplementary Figure 3A and 3B). Moreover, SORCS1 or CUBN knockdown partially reversed the proliferation-suppressive effects (Figure 7E) and abrogated the pro-apoptotic effects (Figure 7F, Supplementary Figure 4A) of miR-431-5p inhibitors. SORCS1 and CUBN siRNAs reversed the suppression of cell proliferation (Figure 7G) and partially reversed the induction of apoptosis in circSPIDR-overexpressing HeLa cells (Figure 7H, Supplementary Figure 4B). These results suggested that SORCS1 and CUBN are direct functional targets of circSPIDR/miR-431-5p signalling.

\section{The clinical value of circSPIDR in human cervical samples}

To explore the clinical value of circSPIDR, we collected an additional 57 normal human cervical tissues and 141 CADC tissues. A qRT-PCR analysis demonstrated that circSPIDR, CUBN and SORCS1 were significantly down-regulated in CADC tissues compared with normal cervical tissues, while miR-431$5 p$ was remarkably upregulated in CADC tissues (Figure 8A). To determine the correlation among these candidate biomarkers, we performed a Pearson's correlation analysis. CircSPIDR levels exhibited a significant inverse correlation with miR-431-5p levels (Figure 8B), but exhibited significant positive correlations with SORCS1 (Figure 8C) and CUBN levels (Figure 8D). The expression of miR-431-5p correlated inversely with the levels of SORCS1 (Figure $8 \mathrm{E}$ ) and $C U B N$ (Figure 8F).

In addition, we performed a receiver operating characteristic (ROC) curve analysis to determine their diagnostic value of these genes in identifying CADC. The area under the ROC curve (AUC, representing the average sensitivity for all possible specificity values) was 0.794 for circSPIDR, 0.787 for miR-431-5p, 0.741 for SORCS1 and 0.711 for $C U B N$, where an AUC $>0.5$ is considered significant (Figure 8G). These data revealed that circSPIDR, miR-431-5p, SORCS1 and CUBN have good potential as diagnostic markers for CADC.

\section{DISCUSSION}

Numerous circRNAs serve as ceRNAs that bind to specific miRNAs and upregualte their target genes, thus promoting tumor development and progression [26-28]. For example, the well-known circRNA CDR1a competitively binds to miR-7 to promote the progression of many cancer types [11, 29-31]. Likewise, circTADA2A sponges miR-203a-3p, thus disinhibiting cyclic adenosine monophosphate responsive element binding protein 3 (CREB3) and increasing the malignant behavior of osteosarcoma [32]. We previously used RNA-sequencing to compare the circRNA, miRNA and mRNA signatures of CADC and normal cervical tissues, in order to identify potential oncogenic and tumor-suppressive circRNAs in CADC $[23,25]$. We proposed that circEYA1 is a ceRNA that binds directly to miR-582-3p to enhance the expression of the miR-582-3p target gene $\mathrm{C}-\mathrm{X}-\mathrm{C}$ motif chemokine ligand 14 (CXCL14) [23]. In the same study, we also identified circSPIDR as a significantly downregulated circRNA in CADC tissues.

CircSPIDR is a novel human circRNA, so its functions had not previously been elucidated. Rat circSPIDR has 
been reported to enhance axon regeneration after sciatic nerve injury partially by altering the phosphoinositide 3-kinase/AKT pathway in dorsal root ganglions [33]. In the present study, we confirmed that circSPIDR was

A
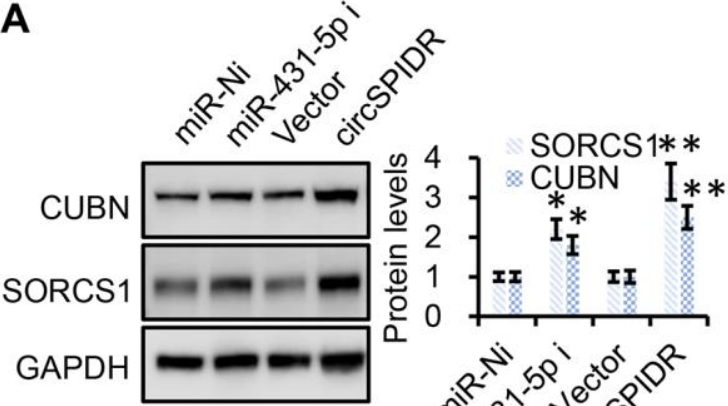

C
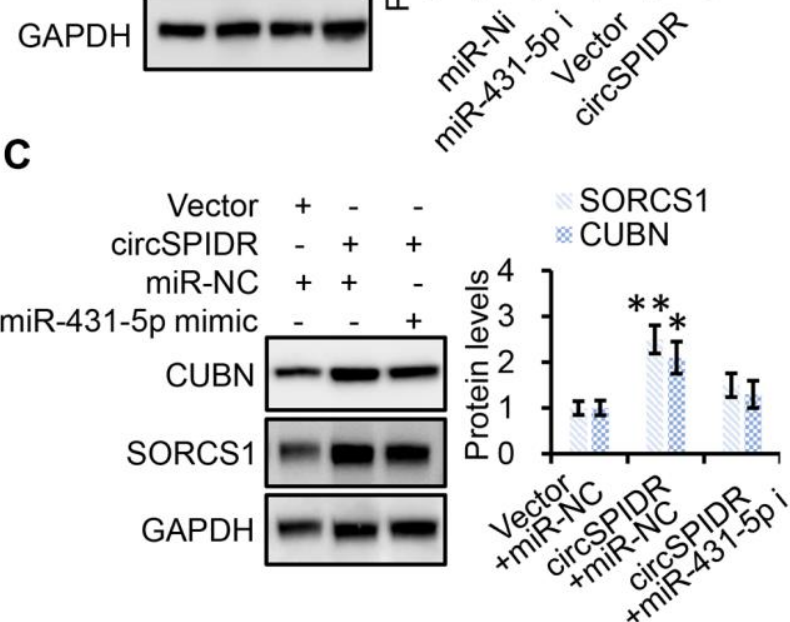

E

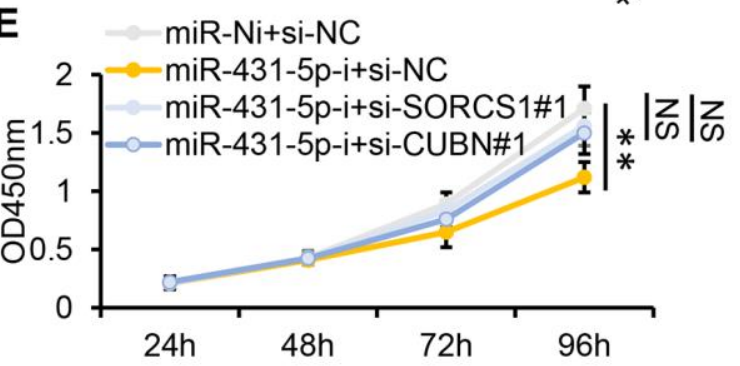

$\mathbf{F}$
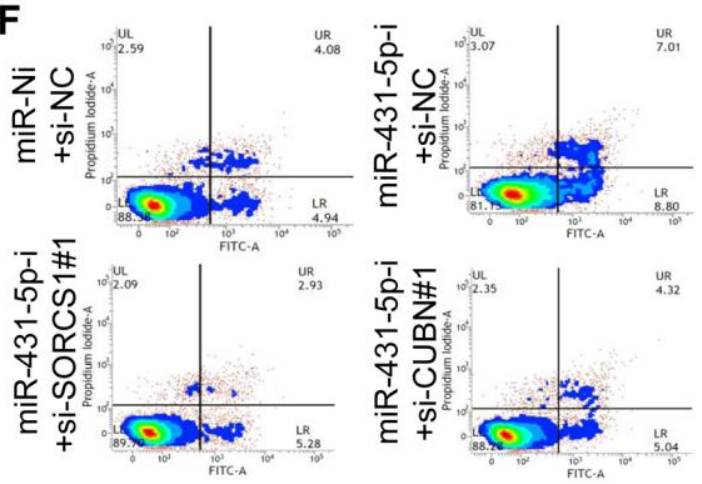

significantly downregulated in CADC tissues. Overexpression of circSPIDR reduced cell viability, inhibited colony formation and promoted apoptosis in CADC cells, while knockdown of circSPIDR had the
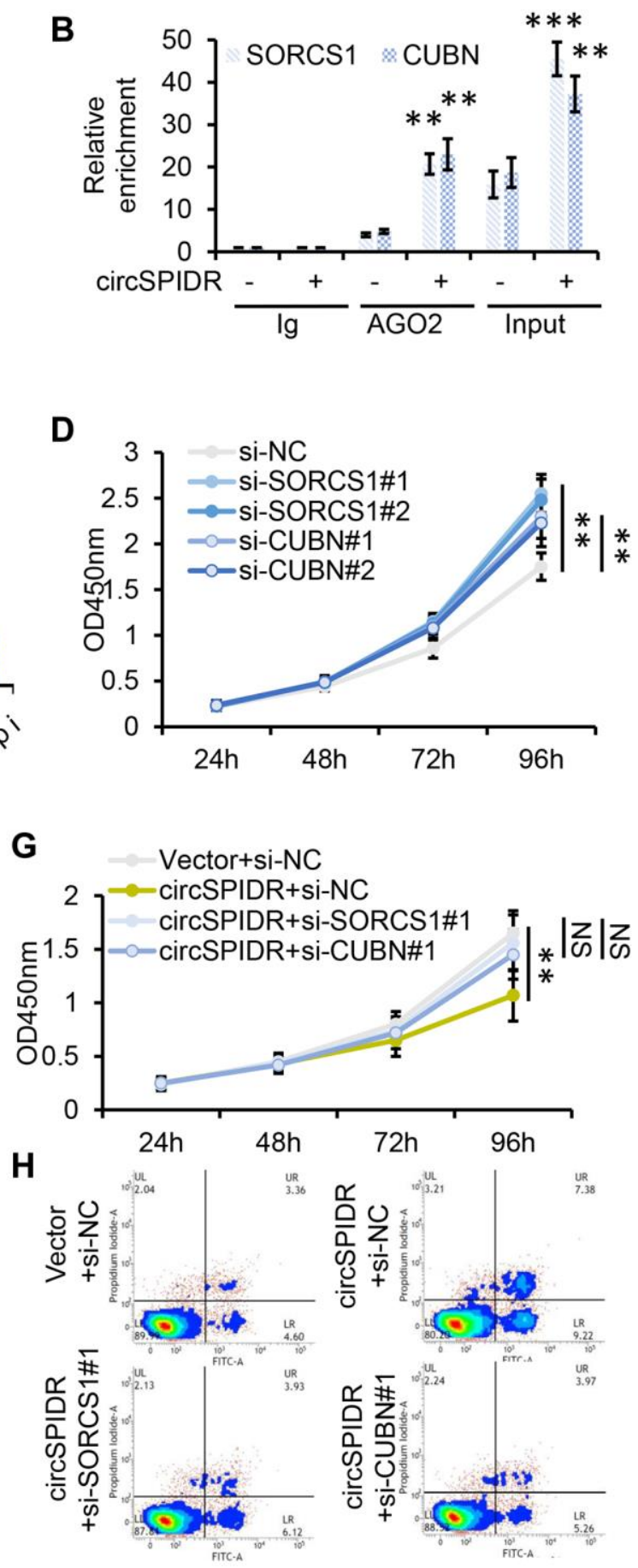

Figure 7. SORCS1 and CUBN are the functional targets of circSPIDR/miR-431-5p signaling. (A) Western blot analysis of SORCS1 and CUBN protein levels in CADC cells transfected with miR-431-5p inhibitors or the circSPIDR expression vector. (B) AGO2-RIP was performed using an anti-AGO2 antibody in HeLa cells transfected with circSPIDR or the vector. Then, qRT-PCR was used to assess and the enrichment of SORCS1 and CUBN. (C) Western blot analysis of SORCS1 and CUBN protein expression in CADC cells co-transfected with circSPIDR and miR431-5p mimics. (D) CCK-8 assay in SORCS1- or CUBN-knockdown HeLa cells. (E, F) CCK-8 assay (E) and apoptosis assay (F) in HeLa cells following miR-431-5p inhibition and SORCS1 or CUBN knockdown. (G, H) CCK-8 assay (G) and apoptosis assay (H) in HeLa cells following circSPIDR overexpression and SORCS1 or CUBN knockdown. NS, not significant; ${ }^{*} P<0.05 ;{ }^{* *} P<0.01 ;{ }^{* * *} P<0.001$. 
opposite effects. CircSPIDR suppressed CADC cell growth both in vitro and in vivo, suggesting that this circRNA is a tumor suppressor in CADC.

To explore the potential ceRNA mechanism of circSPIDR, we performed bioinformatic analyses, which indicated that miR-431-5p shares a binding site with circSPIDR. MiR-431-5p has not been well studied, although it has been proposed as a tumor suppressor in certain cancer types. For instance, miR-431-5p was found to be down-regulated in colon cancer [34] and lung cancer [35], and circ_0001742 was reported to promote tongue squamous cell carcinoma by suppressing miR-431-5p, thus de-repressing activating transcription factor 3 (ATF3) [36]. However, our experiments revealed that, miR-431-5p was significantly up-regulated in CADC tissues, promoted
CADC cell growth and reversed the tumor-suppressive effects of circSPIDR in HeLa cells. Luciferase reporter assays indicated that miR-431-5p reduced the activity of the circSPIDR-wt luciferase reporter, and AGO2-RIP analyses verified that circSPIDR could bind to miR431-5p. These results suggested that circSPIDR bind to miR-431-5p to suppress CADC tumor growth.

We next searched for direct targets of miR-431-5p in HeLa cells, and identified SORCSI and CUBN. SORCS1 is a sorting-related receptor that is involved in metabolic control [37] and has been associated with diabetes in mice and humans [38-41]. SORCS1 was found to be hypermethylated in colorectal cancer tissues, and reduced SORCS1 expression was identified as an independent prognostic factor in colorectal cancer patients [42]. CUBN is an intrinsic factor-vitamin B12
A

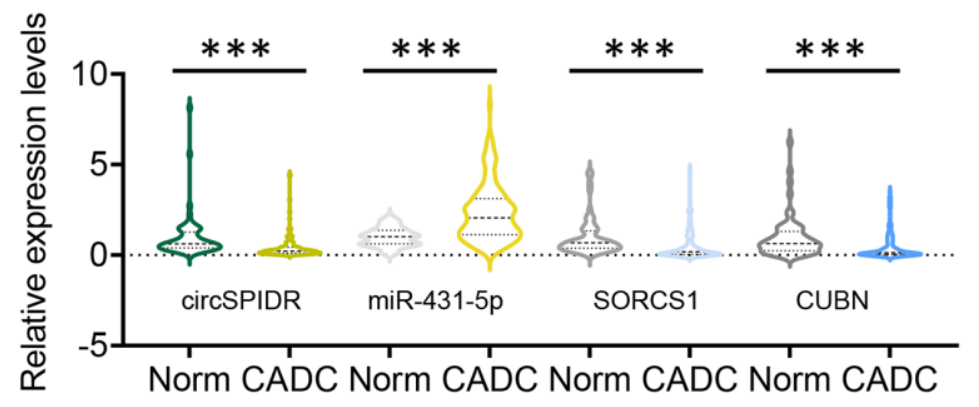

B

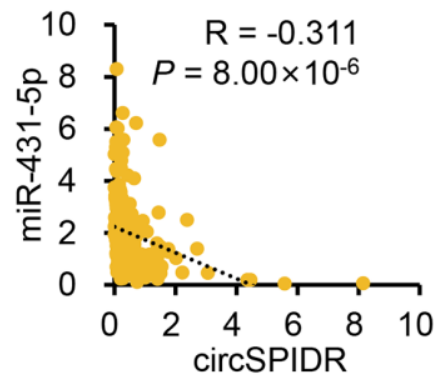

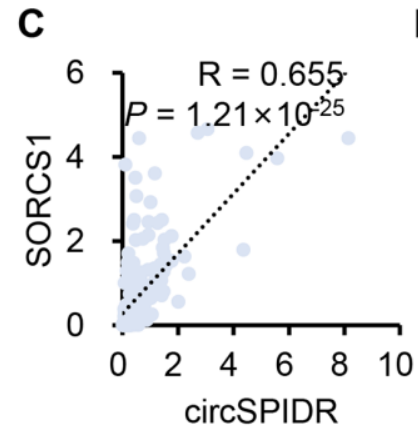

D

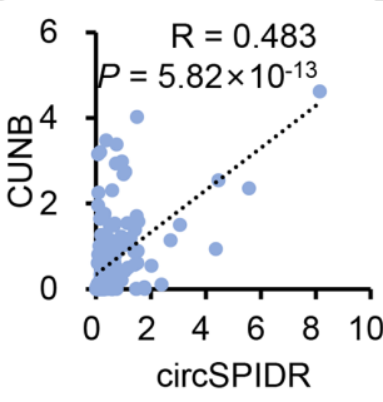

E
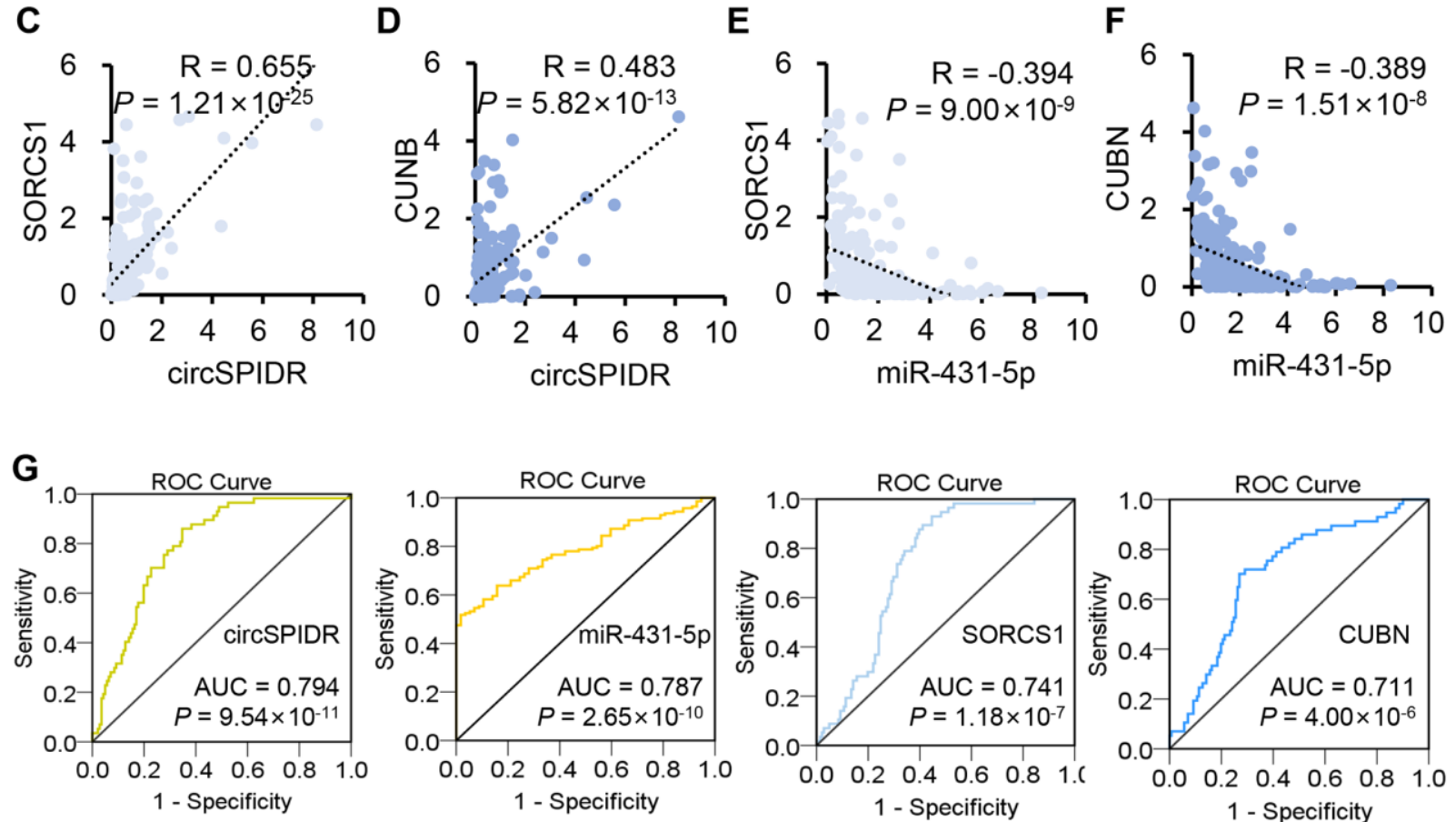

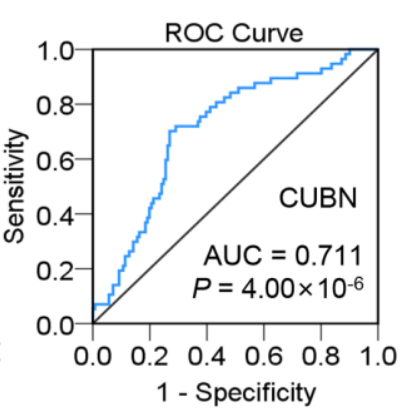

Figure 8. The clinical value of circSPIDR, miR-431-5p, SORCS1 and CUBN expression in CADC tissues. (A) Violin plots showing circSPIDR, miR-431-5p, SORCS1 and CUBN levels determined using qRT-PCR in 57 normal cervical tissues and 141 CADC tissues. (B-D) Pearson correlation analysis between circSPIDR levels and miR-431-5p (B), SORCS1 (C), CUBN (D) levels. (E, F) Pearson correlation analysis between miR-431-5p levels and SORCS1 (E) or CUBN (F) levels. (G) ROC curve analysis of circSPIDR, miR-431-5p, SORCS1 and CUBN detection for CADC diagnosis. ${ }^{* * *} P<0.001$. 
receptor, and mutation of $C U B N$ was found to cause megaloblastic anaemia 1 [43]. Positive CUBN expression was associated with a better prognosis in clear cell renal cell carcinoma patients [44]. Here, we found that SORCS1 and CUBN were down-regulated in CADC tissues, negatively regulated by miR-431-5p and positively regulated by circSPIDR. Correlation analyses indicated that circSPIDR, miR-431-5p, SORCS1 and $C U B N$ levels were tightly correlated in CADC tissues. Moreover, ROC curve analyses suggested that circSPIDR, miR-431-5p, SORCS1 and CUBN expression had good diagnostic potential in CADC patients.

One limitation of our study was that we only used HeLa cells, the currently available CADC cell line. However, we confirmed our observations both in a large cohort of clinical CADC tissues and in animal models, and obtained consistent results. Overall, our findings demonstrated that circSPIDR was significantly downregulated in CADC tissues and could suppress CADC cell growth by competitively binding to miR-431-5p, thus de-repressing SORCS1 and CUBN. Our study has provided insight into circRNA activity in CADC and revealed the potential of circSPIDR as a therapeutic target in this disease.

\section{MATERIALS AND METHODS}

\section{Human tissue specimen collection and ethical approval}

This study was approved by the Ethics Committee of Women's Hospital, Zhejiang University School of Medicine (IRB-2019062-R). Written informed consent was obtained from the patients. The study was performed in accordance with the Declaration of Helsinki. In total, 77 normal cervical tissues and 161 CADC tissues were obtained from Women's Hospital, Zhejiang University School of Medicine from 2009 to 2019. Among them, 40 samples (20 normal cervical tissues and 20 CADC tissues) were used for the initial analysis of circSPIDR and miR431-5p expression, and while the remaining 198 samples were used for qRT-PCR verification of all the candidate genes.

\section{Cell culture}

The human CADC HeLa cell line was purchased from the Institute of Biochemistry and Cell Biology of the Chinese Academy of Sciences (Shanghai, China) and tested negative for mycoplasma contamination. HeLa cells were cultured in Minimum Essential Medium (Cellmax) with $10 \%$ fetal bovine serum (Sijiqing, China).
Plasmids, siRNAs, miRNA mimics and miRNA inhibitors

The circSPIDR expression plasmid, the siRNAs targeting circSPIDR, SORCSI and CUBN, the miR431-5p mimics, the miR-431-5p inhibitors and the relevant negative controls ( $\mathrm{pEX}-3$ empty vector, si-NC, miR-NC, and miR-Ni) were all purchased from GenePharma (Shanghai, China). The circSPIDR overexpression plasmids and siRNAs were transfected into HeLa cells using X-tremeGENE HP DNA transfection reagent (Roche, USA) and DharmaFECT1 transfection reagent (Dharmacon), respectively. The transfection experiments were conducted as described previously [23].

\section{RNA and gDNA extraction, RNase R treatment, RT- PCR, and qRT-PCR}

The RNA and gDNA extraction, RNase R treatment, RT-PCR, and qRT-PCR were performed as previously reported [23]. The primers used in this study are listed in Supplementary Table 1.

\section{Northern blotting}

Total RNA was extracted from the indicated cells and tissues using TRIzol (Invitrogen) and digested with RNase R. Subsequently, a 15- $\mu \mathrm{g}$ sample was loaded onto a $1 \%$ formaldehyde agarose gel and transferred to a Hybond $\mathrm{N}+$ membrane (Amersham) via capillary transfer. Digoxigenin-deoxyuridine triphosphate (DIGdUTP)-labeled circSPIDR probes were generated using a PCR DIG Probe Synthesis Kit (Roche) with the following primers: 5'CCACAGCTAAGTTTCCCAGGAC-3' and 5'TGAGGTGTATGCAAAATGGTCT-3'. The PCR products were gel purified and sequenced prior to hybridization. Hybridization was performed with dUTPlabelled circSPIDR probes in DIG Easy Hyb buffer at $50^{\circ} \mathrm{C}$ overnight. The membrane was then washed and detected in accordance with the instructions for the DIG High Prime DNA Labeling and Detection Starter Kit II (Roche). Finally, the membrane was exposed to X-ray film for $10 \mathrm{~min}$.

\section{CCK-8 and colony formation assays}

CCK-8 and colony formation assays were performed as previously reported [23].

\section{Apoptosis assay}

Treated cells were resuspended in binding buffer containing Annexin V-fluorescein isothiocyanate (FITC) and propidium iodide (PI) for $15 \mathrm{~min}$ in the dark 
(MultiScientces, China). The apoptotic rate was detected with a FACSVerse or FACSCalibur flow cytometer (BD Biosciences).

\section{BrdU assay}

On the basis of the specifications of BrdU (Sigma), HeLa cell proliferation was assessed using a FITCBrdU Cell Proliferation Detection Kit (KGA319-1, KeyGen Biotech, Nanjing, China). In brief, HeLa cells were incubated in a six-well plate and transfected with siRNAs or plasmids. After $48 \mathrm{~h}$ of transfection, $30 \mu \mathrm{M}$ BrdU was added and incubated with the cells for $2 \mathrm{~h}$ at $37^{\circ} \mathrm{C}$. The cells were washed twice with phosphate-buffered saline and subsequently fixed with $4 \%$ paraformaldehyde overnight. Then, the cells were resuspended in $500 \mu \mathrm{L}$ of cell-penetrating fluid for $2 \mathrm{~min}$ at $0^{\circ} \mathrm{C}$ and treated with DNA denaturation working fluid for $30 \mathrm{~min}$ at $4^{\circ} \mathrm{C}$. FITC-anti-BrdU (diluted 1:40) was mixed into the cell plate and incubated with the cells for $30 \mathrm{~min}$ at room temperature. The percentage of BrdU-positive cells was determined using a Flow Cytometer (BD, USA).

\section{Xenograft model}

The animal procedures were approved by the Institutional Animal Care and Use Committee of Zhejiang Chinese Medical University (IACUC20190909-02). All the female BALB/c nude mice used in this study were obtained from Shanghai National Laboratory Animal Center (Shanghai, China). Each 4-week-old nude mouse was subcutaneously injected with $5 \times 10^{6}$ of the indicated cells suspended in $100 \mu \mathrm{L}$ of phosphate-buffered saline ( $n=6 /$ group) or intraperitoneally transplanted with $8 \times 10^{6}$ cells suspended in $150 \mu \mathrm{L}$ phosphatebuffered saline ( $n=5 /$ group). The mice were sacrificed after 42 days, and their tumors were processed for hematoxylin and eosin staining and $\mathrm{Ki}$ 67 detection. The tumor volume was determined as follows: Volume $=\left(\right.$ length $\times$ width $\left.^{2}\right) / 2$.

\section{Luciferase activity assay}

We used pmirGLO dual-luciferase vector (Promega, Madison, WI, USA) to construct different luciferase reporter vectors. We co-transfected $293 \mathrm{~T}$ cells with 50 ng of the corresponding luciferase reporter vector, 50 nm miRNA mimics and $5 \mathrm{ng}$ of a Renilla luciferase reporter vector (pRL-TK, Promega) for $24 \mathrm{~h}$. Luciferase activity was assessed with a dual luciferase reporter assay kit (Promega, E2920). The relative firefly luciferase activity was then normalized to the corresponding Renilla luciferase activity.

\section{RIP}

The RIP experiment was performed with an EZ-Magna RIP Kit (Millipore, Billerica, MA, USA) according to the manufacturer's protocol. An anti-AGO2 antibody (Abcam) was used for the RIP. The purified RNA was confirmed using qRT-PCR analysis.

\section{Fluorescence in situ hybridization}

Cy3-labeled probe sequences for circSPIDR (5'-CY3AUAGAACUUGGCUGAAAGUGUCUUUUGGUAA G-3') and FITC-labeled probe sequences for hsa-miR431-5p (5'-FITC-UGCAUGACGGCCUGCAAGACA3') were synthesized by Sangon Biotech (Shanghai, China) and used to analyze the co-localization of circSPIDR and miR-431-5p in CADC cells. Hybridization was performed overnight using the circSPIDR and miR-431-5p probes, and images were acquired using a Nikon inverted fluorescence microscope (Olympus).

\section{Western blotting}

Total protein were extracted from treated HeLa cells. Then, equal amounts of protein were electrophoretically separated on a $8 \%$ sodium dodecyl sulfate polyacrylamide gel, and transferred to a polyvinylidene difluoride membrane. The membrane was blocked for nonspecific binding, incubated overnight with antibodies against SORCS1 (1:1000, Proteintech, 23002-1-AP), CUBN (1:500, Abcam, ab191073), or GAPDH (1:5000, Santa Cruz, sc-47724), and then incubated with a secondary antibody for $1 \mathrm{~h}$. The bands of proteins were detected using SuperSignal West Pico Chemiluminescent Substrate (Pierce).

\section{Statistical analysis}

All experiments were performed at least three times independently, and images from one representative experiment are shown. Statistical analyses were carried out using SPSS version 24.0 or GraphPad Prism version 9.0. The results are presented as the mean \pm standard deviation. Differences between two groups were evaluated using a two-tailed Student's $t$ test. The correlations among circSPIDR, miR-431-5p, SORCS1 and $C U B N$ levels in CADC patients were assessed using Pearson's correlation analysis. $P$-values $\leq 0.05$ were defined as significant.

\section{AUTHOR CONTRIBUTIONS}

Junfen $\mathrm{Xu}$ and Weiguo Lu conceived and designed this study. Junfen $\mathrm{Xu}$ conducted the experiments and analyzed and checked the data. Weiguo Lu supervised 
the whole project. Junfen $\mathrm{Xu}$ wrote the original draft. Weiguo Lu revised the manuscript. All authors read and approved the final manuscript.

\section{CONFLICTS OF INTEREST}

The authors declare no conflicts of interest related to this study.

\section{FUNDING}

This project was supported by the National Natural Science Foundation of China (Grant No. 82072855) to Doctor Junfen $\mathrm{Xu}$, National Key Research and Development Program of China (Grant No. 2016YFC1302900) to Professor Weiguo Lu, the Fundamental Research Funds for the Central Universities (Grant No. 2019QNA7035 and 2021FZZX001-43) to Doctor Junfen Xu and Innovative Talent Plan of Zhejiang Health Science and Technology Project (Grant No. 2021RC086) to Doctor Junfen Xu.

\section{REFERENCES}

1. Bray F, Ferlay J, Soerjomataram I, Siegel RL, Torre LA, Jemal A. Global cancer statistics 2018: GLOBOCAN estimates of incidence and mortality worldwide for 36 cancers in 185 countries. CA Cancer J Clin. 2018; 68:394-424.

https://doi.org/10.3322/caac.21492

PMID: $\underline{30207593}$

2. Chen W, Zheng R, Baade PD, Zhang S, Zeng H, Bray F, Jemal A, Yu XQ, He J. Cancer statistics in China, 2015. CA Cancer J Clin. 2016; 66:115-32. https://doi.org/10.3322/caac.21338 PMID:26808342

3. Siegel RL, Miller KD, Jemal A. Cancer statistics, 2020. CA Cancer J Clin. 2020; 70:7-30. https://doi.org/10.3322/caac.21590 PMID:31912902

4. Jeck WR, Sharpless NE. Detecting and characterizing circular RNAs. Nat Biotechnol. 2014; 32:453-61.

https://doi.org/10.1038/nbt.2890 PMID:24811520

5. Ashwal-Fluss R, Meyer $M$, Pamudurti NR, Ivanov $A$, Bartok O, Hanan M, Evantal N, Memczak S, Rajewsky $\mathrm{N}$, Kadener S. circRNA biogenesis competes with premRNA splicing. Mol Cell. 2014; 56:55-66. https://doi.org/10.1016/j.molcel.2014.08.019 PMID:25242144

6. Memczak S, Jens M, Elefsinioti A, Torti F, Krueger J, Rybak A, Maier L, Mackowiak SD, Gregersen LH, Munschauer M, Loewer A, Ziebold $U$, Landthaler M, et al. Circular RNAs are a large class of animal RNAs with regulatory potency. Nature. 2013; 495:333-38. https://doi.org/10.1038/nature11928 PMID:23446348

7. Wilusz JE. A $360^{\circ}$ view of circular RNAs: From biogenesis to functions. Wiley Interdiscip Rev RNA. 2018; 9:e1478. https://doi.org/10.1002/wrna.1478 PMID:29655315

8. Gao Y, Wang J, Zheng Y, Zhang J, Chen S, Zhao F. Comprehensive identification of internal structure and alternative splicing events in circular RNAs. Nat Commun. 2016; 7:12060.

https://doi.org/10.1038/ncomms12060 PMID:27350239

9. Guarnerio J, Bezzi M, Jeong JC, Paffenholz SV, Berry K, Naldini MM, Lo-Coco F, Tay Y, Beck AH, Pandolfi PP. Oncogenic Role of Fusion-circRNAs Derived from Cancer-Associated Chromosomal Translocations. Cell. 2016; 165:289-302. https://doi.org/10.1016/j.cell.2016.03.020 PMID:27040497

10. Lasda E, Parker R. Circular RNAs: diversity of form and function. RNA. 2014; 20:1829-42. https://doi.org/10.1261/rna.047126.114 PMID:25404635

11. Li C, Li M, Xue Y. Downregulation of CircRNA CDR1as specifically triggered low-dose Diosbulbin-B induced gastric cancer cell death by regulating miR-7-5p/REG $\gamma$ axis. Biomed Pharmacother. 2019; 120:109462.

https://doi.org/10.1016/j.biopha.2019.109462 PMID:31542615

12. Li Y, Zhang J, Pan S, Zhou J, Diao X, Liu S. CircRNA CDR1as knockdown inhibits progression of non-smallcell lung cancer by regulating miR-219a-5p/SOX5 axis. Thorac Cancer. 2020; 11:537-48. https://doi.org/10.1111/1759-7714.13274 PMID: $\underline{31917898}$

13. Yuan W, Zhou R, Wang J, Han J, Yang X, Yu H, Lu H, Zhang X, Li P, Tao J, Wei J, Lu Q, Yang H, Gu M. Circular RNA Cdr1as sensitizes bladder cancer to cisplatin by upregulating APAF1 expression through miR-1270 inhibition. Mol Oncol. 2019; 13:1559-76.

https://doi.org/10.1002/1878-0261.12523 PMID:31131537

14. Huang X, Li Z, Zhang Q, Wang W, Li B, Wang L, Xu Z, Zeng A, Zhang X, Zhang X, He Z, Li Q, Sun G, et al. Circular RNA AKT3 upregulates PIK3R1 to enhance cisplatin resistance in gastric cancer via miR-198 suppression. Mol Cancer. 2019; 18:71. https://doi.org/10.1186/s12943-019-0969-3 PMID: $\underline{30927924}$ 
15. Xia X, Li X, Li F, Wu X, Zhang $M$, Zhou $H$, Huang $N$, Yang $X$, Xiao F, Liu D, Yang L, Zhang N. A novel tumor suppressor protein encoded by circular AKT3 RNA inhibits glioblastoma tumorigenicity by competing with active phosphoinositide-dependent Kinase-1. Mol Cancer. 2019; 18:131.

https://doi.org/10.1186/s12943-019-1056-5

PMID:31470874

16. Garikipati VNS, Verma SK, Cheng Z, Liang D, Truongcao MM, Cimini M, Yue Y, Huang G, Wang C, Benedict C, Tang Y, Mallaredy V, Ibetti J, et al. Circular RNA CircFndc3b modulates cardiac repair after myocardial infarction via FUS/VEGF-A axis. Nat Commun. 2019; 10:4317.

https://doi.org/10.1038/s41467-019-11777-7 PMID:31541092

17. Legnini I, Di Timoteo G, Rossi F, Morlando M, Briganti F, Sthandier O, Fatica A, Santini T, Andronache A, Wade M, Laneve P, Rajewsky N, Bozzoni I. CircZNF609 Is a Circular RNA that Can Be Translated and Functions in Myogenesis. Mol Cell. 2017; 66:2237.e9.

https://doi.org/10.1016/j.molcel.2017.02.017 PMID:28344082

18. Rossi F, Legnini I, Megiorni F, Colantoni A, Santini T, Morlando M, Di Timoteo G, Dattilo D, Dominici C, Bozzoni I. Circ-ZNF609 regulates G1-S progression in rhabdomyosarcoma. Oncogene. 2019; 38:3843-54. https://doi.org/10.1038/s41388-019-0699-4 PMID:30670781

19. Pamudurti NR, Bartok O, Jens M, Ashwal-Fluss R, Stottmeister C, Ruhe L, Hanan M, Wyler E, PerezHernandez D, Ramberger E, Shenzis S, Samson M, Dittmar G, et al. Translation of CircRNAs. Mol Cell. 2017; 66:9-21.e7.

https://doi.org/10.1016/i.molcel.2017.02.021 PMID:28344080

20. Meng S, Zhou H, Feng Z, Xu Z, Tang Y, Li P, Wu M. CircRNA: functions and properties of a novel potential biomarker for cancer. Mol Cancer. 2017; 16:94. https://doi.org/10.1186/s12943-017-0663-2 PMID:28535767

21. Tang W, Fu K, Sun H, Rong D, Wang H, Cao H. CircRNA microarray profiling identifies a novel circulating biomarker for detection of gastric cancer. Mol Cancer. 2018; 17:137.

https://doi.org/10.1186/s12943-018-0888-8 PMID:30236115

22. Zhang HD, Jiang LH, Hou JC, Zhong SL, Zhou SY, Zhu LP, Li J, Wang DD, Sun DW, Ji ZL, Tang JH. Circular RNA hsa_circ_0052112 promotes cell migration and invasion by acting as sponge for miR-125a-5p in breast cancer. Biomed Pharmacother. 2018; 107:1342-53. https://doi.org/10.1016/j.biopha.2018.08.030 PMID:

23. Xu J, Zhang $Y$, Huang $Y$, Dong $X$, Xiang $Z$, Zou J, Wu L, Lu W. circEYA1 Functions as a Sponge of miR-582-3p to Suppress Cervical Adenocarcinoma Tumorigenesis via Upregulating CXCL14. Mol Ther Nucleic Acids. 2020; 22:1176-90. https://doi.org/10.1016/j.omtn.2020.10.026 PMID:33312754

24. Hansen TB, Jensen TI, Clausen BH, Bramsen JB, Finsen $B$, Damgaard CK, Kjems J. Natural RNA circles function as efficient microRNA sponges. Nature. 2013; 495:384-88.

https://doi.org/10.1038/nature11993

PMID:23446346

25. $\mathrm{Xu} \mathrm{J,} \mathrm{Zou} \mathrm{J,} \mathrm{Wu} \mathrm{L,} \mathrm{Lu} \mathrm{W.} \mathrm{Transcriptome} \mathrm{analysis}$ uncovers the diagnostic value of miR-192-5p/HNF1AAS1/VIL1 panel in cervical adenocarcinoma. Sci Rep. 2020; 10:16584.

https://doi.org/10.1038/s41598-020-73523-0

PMID:33024199

26. Piwecka $M$, Glažar $P$, Hernandez-Miranda $L R$, Memczak S, Wolf SA, Rybak-Wolf A, Filipchyk A, Klironomos F, Cerda Jara CA, Fenske $P$, Trimbuch T, Zywitza $\mathrm{V}$, Plass $\mathrm{M}$, et al. Loss of a mammalian circular RNA locus causes miRNA deregulation and affects brain function. Science. 2017; 357:eaam8526.

https://doi.org/10.1126/science.aam8526 PMID:28798046

27. Zheng Q, Bao C, Guo W, Li S, Chen J, Chen B, Luo Y, Lyu D, Li Y, Shi G, Liang L, Gu J, He X, Huang S. Circular RNA profiling reveals an abundant circHIPK3 that regulates cell growth by sponging multiple miRNAs. Nat Commun. 2016; 7:11215. https://doi.org/10.1038/ncomms11215 PMID:27050392

28. Chen LL. The biogenesis and emerging roles of circular RNAs. Nat Rev Mol Cell Biol. 2016; 17:205-11. https://doi.org/10.1038/nrm.2015.32 PMID:26908011

29. Tang W, Ji M, He G, Yang L, Niu Z, Jian M, Wei Y, Ren $\mathrm{L}, \mathrm{Xu}$ J. Silencing CDR1as inhibits colorectal cancer progression through regulating microRNA-7. Onco Targets Ther. 2017; 10:2045-56. https://doi.org/10.2147/OTT.S131597 PMID:28435295

30. Zhong Q, Huang J, Wei J, Wu R. Circular RNA CDR1as sponges miR-7-5p to enhance E2F3 stability and promote the growth of nasopharyngeal carcinoma. Cancer Cell Int. 2019; 19:252. https://doi.org/10.1186/s12935-019-0959-y PMID:31582908 
31. Yu L, Gong X, Sun L, Zhou Q, Lu B, Zhu L. The Circular RNA Cdr1as Act as an Oncogene in Hepatocellular Carcinoma through Targeting miR-7 Expression. PLoS One. 2016; 11:e0158347.

https://doi.org/10.1371/journal.pone.0158347 PMID:27391479

32. Wu Y, Xie Z, Chen J, Chen J, Ni W, Ma Y, Huang K, Wang G, Wang J, Ma J, Shen S, Fan S. Circular RNA circTADA2A promotes osteosarcoma progression and metastasis by sponging miR-203a-3p and regulating CREB3 expression. Mol Cancer. 2019; 18:73. https://doi.org/10.1186/s12943-019-1007-1 PMID:30940151

33. Mao S, Huang T, Chen Y, Shen L, Zhou S, Zhang S, Yu B. Circ-Spidr enhances axon regeneration after peripheral nerve injury. Cell Death Dis. 2019; 10:787. https://doi.org/10.1038/s41419-019-2027-x PMID:31624232

34. Huang W, Zeng C, Hu S, Wang L, Liu J. ATG3, a Target of miR-431-5p, Promotes Proliferation and Invasion of Colon Cancer via Promoting Autophagy. Cancer Manag Res. 2019; 11:10275-85. https://doi.org/10.2147/CMAR.S226828 PMID:31849517

35. Jiang Q, Cheng L, Ma D, Zhao Y. FBXL19-AS1 exerts oncogenic function by sponging miR-431-5p to regulate RAF1 expression in lung cancer. Biosci Rep. 2019; 39:BSR20181804. https://doi.org/10.1042/BSR20181804 PMID:30610161

36. Hu YT, Li XX, Zeng LW. Circ_0001742 promotes tongue squamous cell carcinoma progression via miR431-5p/ATF3 axis. Eur Rev Med Pharmacol Sci. 2019; 23:10300-12.

https://doi.org/10.26355/eurrev 201912_19668 PMID:31841185

37. Hermey G, Riedel IB, Hampe W, Schaller HC, Hermans-Borgmeyer I. Identification and characterization of SorCS, a third member of a novel receptor family. Biochem Biophys Res Commun. 1999; 266:347-51.

https://doi.org/10.1006/bbrc.1999.1822

PMID: 10600506

38. Subkhangulova A, Malik AR, Hermey G, Popp O, Dittmar G, Rathjen T, Poy MN, Stumpf A, Beed PS, Schmitz D, Breiderhoff T, Willnow TE. SORCS1 and SORCS3 control energy balance and orexigenic peptide production. EMBO Rep. 2018; 19:e44810. https://doi.org/10.15252/embr.201744810 PMID:29440124

39. Clee SM, Yandell BS, Schueler KM, Rabaglia ME, Richards OC, Raines SM, Kabara EA, Klass DM, Mui ET,
Stapleton DS, Gray-Keller MP, Young MB, Stoehr JP, et al. Positional cloning of Sorcs1, a type 2 diabetes quantitative trait locus. Nat Genet. 2006; 38:688-93. https://doi.org/10.1038/ng1796 PMID:16682971

40. Goodarzi MO, Lehman DM, Taylor KD, Guo X, Cui J, Quiñones MJ, Clee SM, Yandell BS, Blangero J, Hsueh WA, Attie AD, Stern MP, Rotter JI. SORCS1: a novel human type 2 diabetes susceptibility gene suggested by the mouse. Diabetes. 2007; 56:1922-29.

https://doi.org/10.2337/db06-1677

PMID: $\underline{17426289}$

41. Paterson $A D$, Waggott $D$, Boright $A P$, Hosseini $S M$, Shen E, Sylvestre MP, Wong I, Bharaj B, Cleary PA, Lachin JM, Below JE, Nicolae D, Cox NJ, et al, and MAGIC (Meta-Analyses of Glucose and Insulin-related traits Consortium), and Diabetes Control and Complications Trial/Epidemiology of Diabetes Interventions and Complications Research Group. A genome-wide association study identifies a novel major locus for glycemic control in type 1 diabetes, as measured by both A1C and glucose. Diabetes. 2010; 59:539-49.

https://doi.org/10.2337/db09-0653

PMID: $\underline{19875614}$

42. Huang $P Z$, Peng SY, Yu HC, Huang $L$, Yao $Q$, Wang $X L$, Tan SY, Zhou JM, Wang PN, Huang AP, Bai LL, Luo YX, Huang MJ. Decreased expression of SorCS1 in colorectal cancer: An independent predictor of poor prognosis. Neoplasma. 2020; 67:119-28. https://doi.org/10.4149/neo 2019 190221N146 PMID: $\underline{31829024}$

43. Aminoff $M$, Carter JE, Chadwick RB, Johnson C, Gräsbeck R, Abdelaal MA, Broch $\mathrm{H}$, Jenner LB, Verroust PJ, Moestrup SK, de la Chapelle A, Krahe R. Mutations in CUBN, encoding the intrinsic factor-vitamin B12 receptor, cubilin, cause hereditary megaloblastic anaemia 1. Nat Genet. 1999; 21:309-13.

https://doi.org/10.1038/6831

PMID: 10080186

44. Gremel G, Djureinovic D, Niinivirta $M$, Laird A, Ljungqvist $\mathrm{O}$, Johannesson $\mathrm{H}$, Bergman J, Edqvist $\mathrm{PH}$, Navani S, Khan N, Patil T, Sivertsson $\AA$, Uhlén $M$, et al. A systematic search strategy identifies cubilin as independent prognostic marker for renal cell carcinoma. BMC Cancer. 2017; 17:9. https://doi.org/10.1186/s12885-016-3030-6

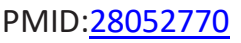




\section{SUPPLEMENTARY MATERIALS}

\section{Supplementary Figures}

A

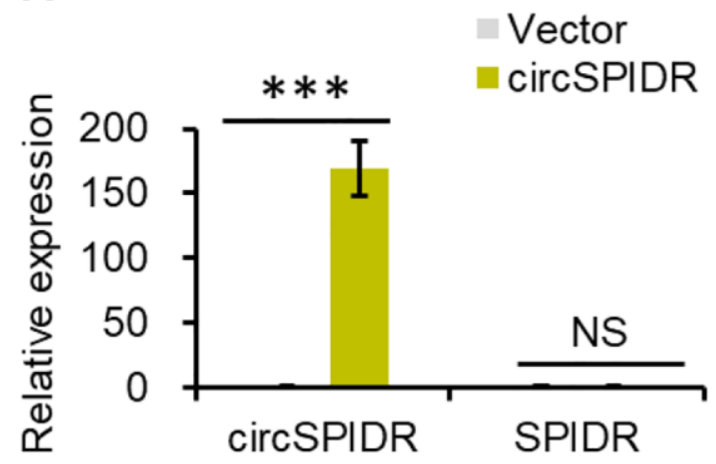

B

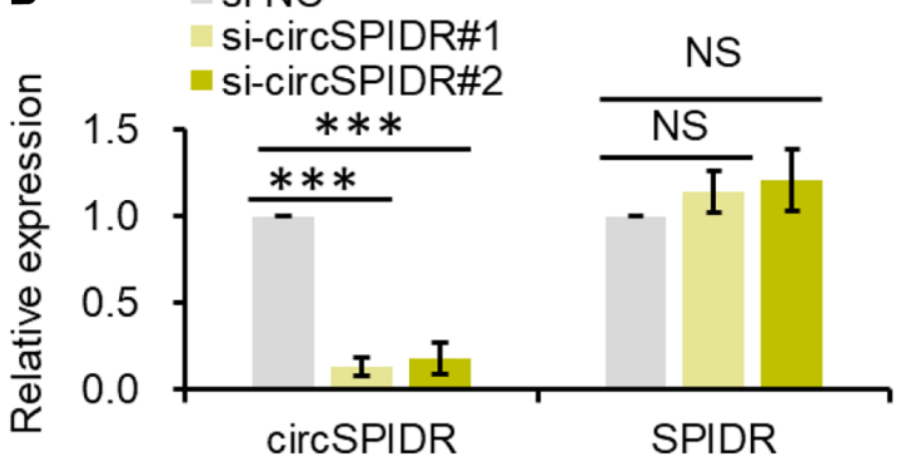

Supplementary Figure 1. Transfection efficiency of circSPIDR expression vector and siRNAs in HeLa cells. (A) The expression level of circSPIDR in HeLa cells after transfection with circSPIDR expression vector was detected by qRT-PCR. Vector was taken as empty vector control. (B) The expression level of circSPIDR in HeLa cells after transfection with circSPIDR siRNA\#1 or \#2 was detected by qRT-PCR.siNC was taken as negative siRNA control. NS, not significant; ${ }^{* * *} P<0.001$.
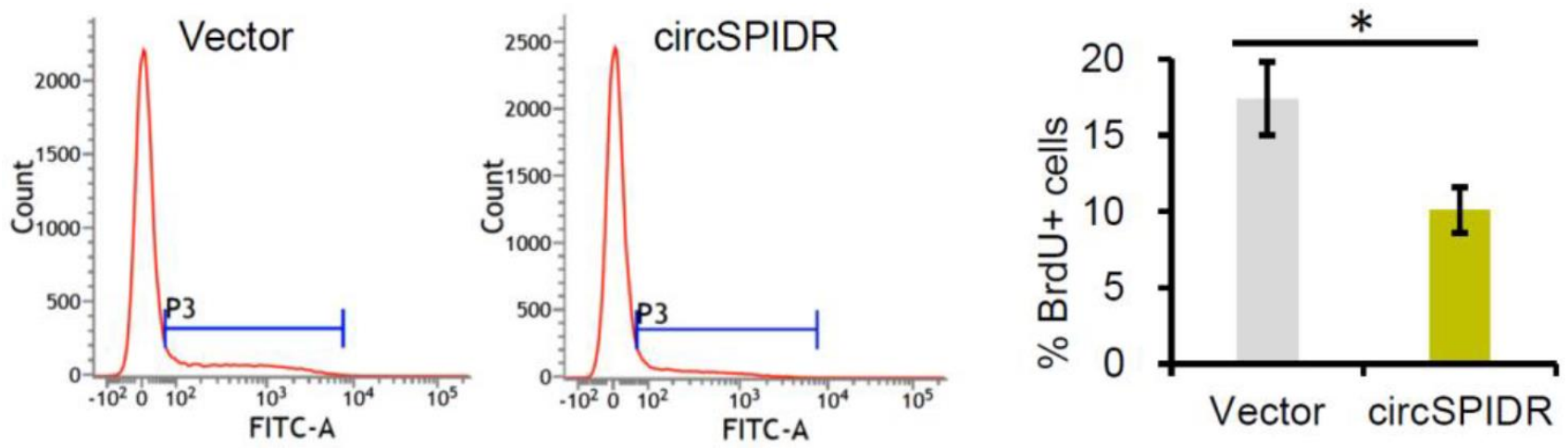

Supplementary Figure 2. Overexpression of circSPIDR suppressed cell proliferation in HeLa cells by BrdU assay. The percentage of BrdU positive cells was shown. ${ }^{*} P<0.05$. 
A
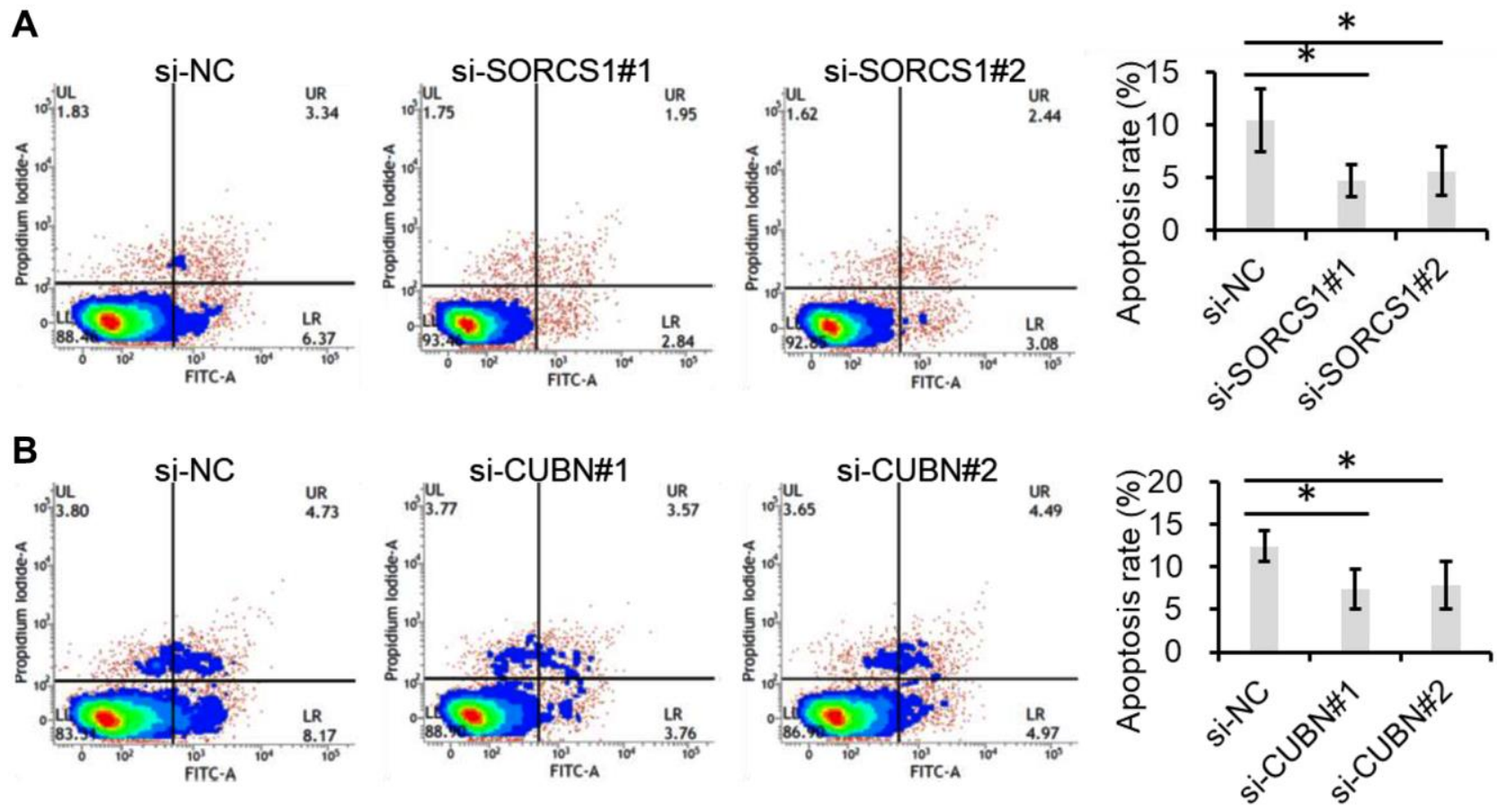

Supplementary Figure 3. Knockdown of SORCS1 and CUBN decreased cell apoptosis in HeLa cells. (A) Cell apoptosis assay for HeLa cells with SORCS1 knockdown. (B) Cell apoptosis assay for HeLa cells with CUBN knockdown. ${ }^{*} P<0.05$.

A

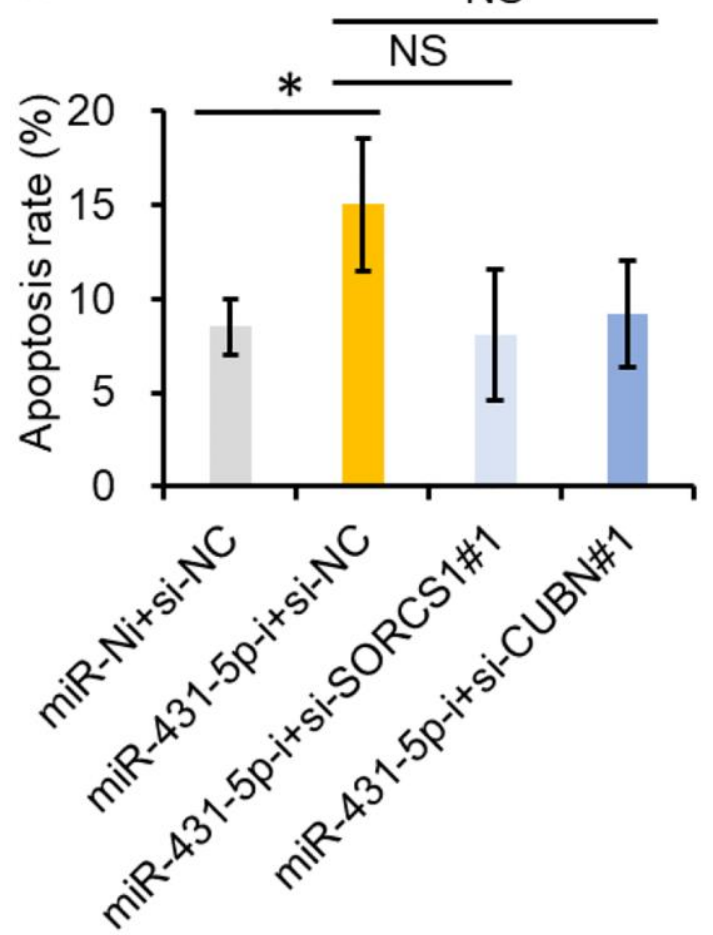

B
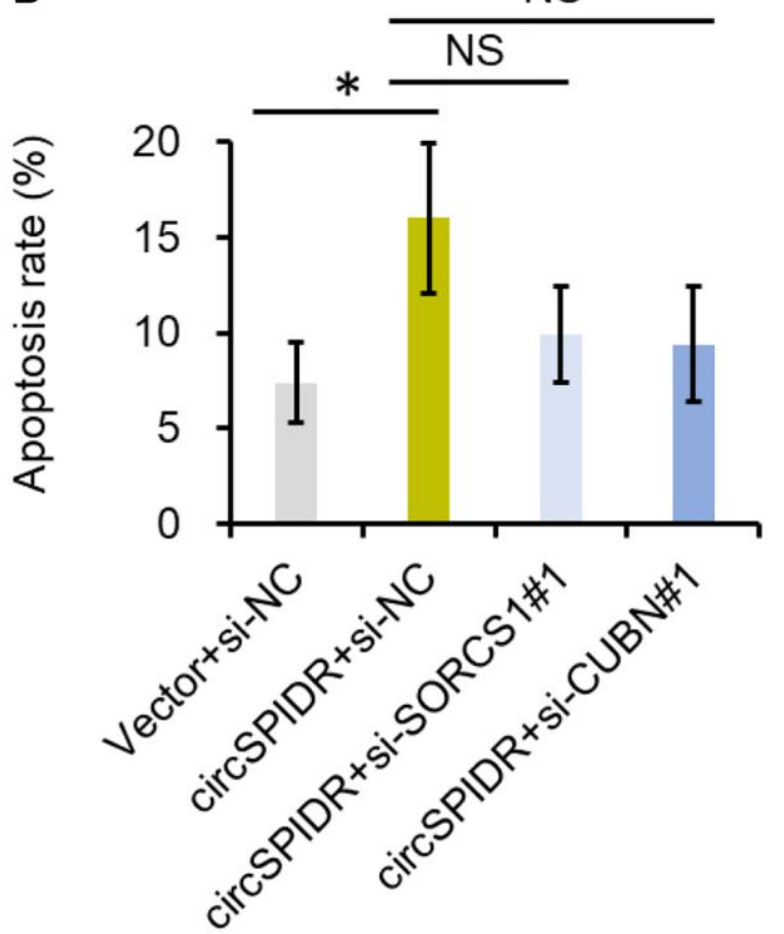

Supplementary Figure 4. miR-431-5p and circSPIDR contribute to cell apoptosis via targeting SORCS1 and CUBN. (A) Cell apoptosis assay for HeLa cells with miR-431-5p inhibitors and SORCS1 knockdown or CUBN knockdown. (B) Cell apoptosis assay for HeLa cells with circSPIDR and SORCS1 knockdown or CUBN knockdown. NS, not significant; ${ }^{*} P<0.05$. 


\section{Supplementary Table}

Supplementary Table 1. The primer sequences used in this study.

\begin{tabular}{|c|c|c|}
\hline Primers & Strand & Sequences $\left(5^{\prime}-3^{\prime}\right)$ \\
\hline circSPIDR & Forward & AAGACACTTTCAGCCAAGTTC \\
\hline (divergent primers) & Reverse & CTCTCCATAATCTGTCTTGC \\
\hline circSPIDR & Forward & ATTCAGAATCCCCTCACA \\
\hline (convergent primers) & Reverse & CTGTTGATTTTGTCCTTG \\
\hline \multirow[t]{2}{*}{ SPIDR } & Forward & ACTGGTGTGATCGTGTCTCTG \\
\hline & Reverse & CGTGCACATTACCCAGCCTA \\
\hline \multirow[t]{2}{*}{ ADD2 } & Forward & CTCATTTCCTCCCAGCCTCG \\
\hline & Reverse & GCCGCAGTTCCTTGACAAAA \\
\hline \multirow[t]{2}{*}{ CDHR1 } & Forward & TAATTGGTCTCGTCAGGCGG \\
\hline & Reverse & GGGAGGGCAGAGACCACAAT \\
\hline \multirow[t]{2}{*}{ CUBN } & Forward & AGGAACTGAGGAGGTGGACA \\
\hline & Reverse & GACTGGAAGACGGCAGTGAA \\
\hline \multirow[t]{2}{*}{ DIRAS2 } & Forward & GAAGCTCTGAGCGGAGTTGT \\
\hline & Reverse & AAACCTCAACACCAGGGAGC \\
\hline \multirow[t]{2}{*}{ EFCAB1 } & Forward & AGTAACTGAGACCTCACCGC \\
\hline & Reverse & TGTCTGGCGCTCAGAGAATC \\
\hline \multirow[t]{2}{*}{ KLF8 } & Forward & TGTGGTATCTCCTCGTGGGT \\
\hline & Reverse & GGTGGGGGCTATGAAAACCA \\
\hline \multirow[t]{2}{*}{ L3MBTL4 } & Forward & GTAGCTCGGTCGGCGTTG \\
\hline & Reverse & TTGGGCTGTTTCATTGCCAC \\
\hline \multirow[t]{2}{*}{ NXPH3 } & Forward & GACCCCGAAAAGAGAAGGGG \\
\hline & Reverse & ATAGAGGCTACCCTGCACCA \\
\hline \multirow[t]{2}{*}{ PLEKHG7 } & Forward & AAATTACCAGCTTCAGGGGCT \\
\hline & Reverse & TGTGAAAAGTTCCCACACGG \\
\hline \multirow[t]{2}{*}{ SCN3B } & Forward & GATTCCAGTCGGAACGCAAC \\
\hline & Reverse & GGGTAAGCTCAGCTCGGAAG \\
\hline \multirow[t]{2}{*}{ SHE } & Forward & TGTGACCCTCAGGAAGGTTAAG \\
\hline & Reverse & TCCTCTTCCAGTCTGCACCA \\
\hline \multirow[t]{2}{*}{ SORCS1 } & Forward & CTGCCGGCATTTTGGTTCAA \\
\hline & Reverse & ACCACCTTCCTGTACCCAGT \\
\hline \multirow[t]{2}{*}{ SOX5 } & Forward & AGGTAGCCATGGTGACAAGC \\
\hline & Reverse & ACAAGTCTCTTGCGTCAGCA \\
\hline \multirow[t]{2}{*}{ TMEM213 } & Forward & CTCAACGTGGACTTCTGCCCA \\
\hline & Reverse & CATCAGTTTGTCCACACAGAGC \\
\hline \multirow[t]{2}{*}{ WASF3 } & Forward & TGAGCCAAAGTGGTGATGCT \\
\hline & Reverse & CGGGGCTCAATGTTCCTCTT \\
\hline \multirow[t]{2}{*}{ ZNF483 } & Forward & GCTCTACGCAATCCTAGCCA \\
\hline & Reverse & AGTTATGTGCTCCACACAGCA \\
\hline \multirow[t]{2}{*}{ si-circSPIDR\#1 } & Forward & AAAAGACACUUUCAGCCAATT \\
\hline & Reverse & UUGGCUGAAAGUGUCUUUUTT \\
\hline \multirow[t]{2}{*}{ si-circSPIDR\#2 } & Forward & CACUUUCAGCCAAGUUCUATT \\
\hline & Reverse & UAGAACUUGGCUGAAAGUGTT \\
\hline \multirow[t]{2}{*}{ si-SORCS1\#1 } & Forward & CCAACAAGCGUAAGAUAAUTT \\
\hline & Reverse & AUUAUCUUACGCUUGUUGGTT \\
\hline
\end{tabular}




\begin{tabular}{cll} 
si-SORCS1\#2 & Forward & GCUGCCCUAUUGCUCACUATT \\
& Reverse & UAGUGAGCAAUAGGGCAGCTT \\
si-CUBN\#1 & Forward & GGAAUAUUCUAUGGCUCAATT \\
& Reverse & UUGAGCCAUAGAAUAUUCCTT \\
si-CUBN\#2 & Forward & GCAGACUACCCAAAUGAUATT \\
& Reverse & UAUCAUUUGGGUAGUCUGCTT \\
\hline
\end{tabular}

